\title{
3D Segmentation for Super-Resolution Imaging of Bacterial Biofilms
}

\author{
A Thesis \\ Presented to
}

the faculty of the School of Engineering and Applied Science

University of Virginia

\author{
in partial fulfillment \\ of the requirements for the degree \\ Master of Science \\ by \\ Jie Wang \\ May 2017
}




\title{
APPROVAL SHEET
}

\author{
This Thesis \\ is submitted in partial fulfillment of the requirements \\ for the degree of \\ Master of Science
}

Author Signature:

Jie Wang

This Thesis has been read and approved by the examining committee:

Advisor: Scott T. Acton

Committee Member: Zongli Lin

Committee Member: Andreas Gahlmann

Committee Member:

Committee Member:

Committee Member:

Accepted for the School of Engineering and Applied Science:

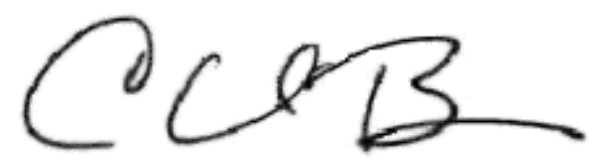

Craig H. Benson, School of Engineering and Applied Science

May 2017 


\section{D Segmentation for Super-Resolution Imaging of Bacterial Biofilms}

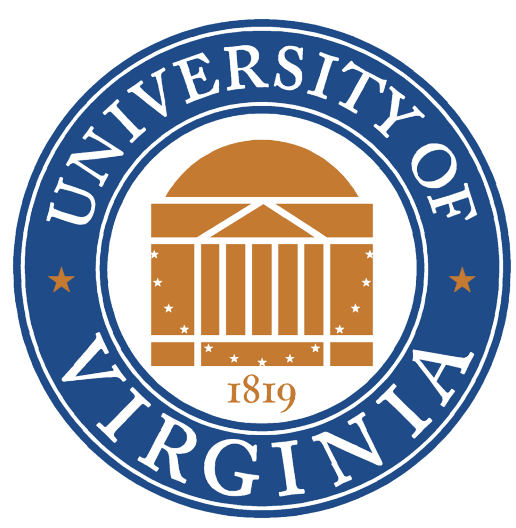

A Thesis

Presented to the Faculty of School of Engineering and Applied Science

In Partial Fulfillment of the Requirements for the Master of Science Degree in Electrical Engineering

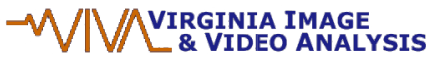

Advisor: Prof. Scott T. Acton

By

JIE WANG

MAY 2017 

he emergence of super-resolution microscopy using lattice light sheet is enabling the exploration of structure and function in living tissues organization. Such a technique overcomes the diffraction limit in traditional confocal microscopy, which also provides non-phototoxic three-dimensional images at resolutions ten times smaller than that provided by traditional light microscopy. Unfortunately, the standard tools used in the image analysis community to perform segmentation and other higher-level analyses cannot be applied naïvely to these data.

In this thesis, Bact-3D is presented for segmenting super-resolution images of multi-leveled, living bacteria cultured in vitro. It incorporates a novel initialization approach that exploits the geometry of the bacterial cells as well as iterative local level set evolution that is tailored to the biological application. In experiments where segmentation is used as precursor to cell detection, the Bact-3D matches or improves upon the Dice score and mean-squared error of three existing methods, while yielding a substantial improvement in cell detection accuracy. In addition to providing improvements in performance over the state-of-the-art, this report also characterizes the tradeoff between imaging resolution and segmentation quality. Evaluation of the algorithm complexity and operating times of Bact-3D as well as alternative solutions are also discussed.

Improvements based on Bact-3D method are proposed to increase the applicability in extended datasets, where bacteria are randomly distributed and densely overlapped. The improved methodology ensures the locality 
of 3D level set based active surface model, which is achieved by proposing intuitive distance velocity field (DVF), of the level set evolution. Preliminary experimental results that compare the performance of improved approach with two existing methods exhibit competitive advantage in segmenting neighboring cells. Comprehensive application of combining geometric and parametric segmentation techniques on these challenging datasets is also proposed for future work. 


\section{DEDICATION AND ACKNOWLEDGEMENTS}

To My Loving Family and Friends.

I am so grateful to my advisor, Professor Scott T. Acton, who led me to the field of image processing two years ago and allowed me to join VIVA lab to proceed my Master's research in this past one and half year. I feel so lucky for being in VIVA lab under the support of this nice professor, who kindly protects his students all the time.

I would also thank the support from Professor Andreas Gahlmann and his lab. He changed my understanding of bacteria and showed me the great potential of bacterial biofilms. These made my research in this thesis applicable and significant.

I would like to thank Professor Zongli Lin for sparing time to chair my committee and to offer me advices. Also, I would like to thank him for his tutoring in my Introduction of Control Systems class.

I would like to express my grateful to Andrea who spent a long time to help me revise this thesis. I would like to thank him and Ritu for helping me with the project and with the paper for ICIP.

I would also send my strong gratitude to everyone in the VIVA lab and VITAL lab in Rice 328. They are so nice and generous. Thank you so much! You are the best group!

I would never forget my best roommates, Pingping and Xiao. I would like to thank them for picking me up from school every day in this semester at no matter what time I finished my work to support my research. 
Finally, my dear family, my dear father and mother, I have thousands of words for you! In short, it is three words and eight letters, "I love you".

Thank you all the way. 


\section{AUTHOR's DECLARATION}

I

declare that the work in this thesis was carried out in accordance with the requirements of University's Regulations and Code of Practice for in the text, the work is the candidate's own work. Work done in collaboration with, or with the assistance of, others, is indicated as such. Any views expressed in the dissertation are those of the author.

SIGNED: Jie Wang

DATE:

$05 / 2017$ 



\section{TABLe OF Contents}

\section{Page}

List of Tables $\quad$ ix

List of Figures $\quad$ xi

1 Introduction 1

1.1 Bacteria and biofilm basics . . . . . . . . . . . . . . 3

1.2 Imaging techniques $\ldots \ldots \ldots \ldots \ldots$

1.3 Segmentation techniques overview . . . . . . . . . . . 6

1.4 Thesis overview . . . . . . . . . . . . . . 7

2 Segmentation Background $\quad 9$

2.1 Parametric active contours . . . . . . . . . . . . . . 9

2.2 Geometric active contours . . . . . . . . . . . . . . . 12

2.2.1 General expression . . . . . . . . . . . . . . . . 12

2.2 .2 Active contours without edges . . . . . . . . . . 14

2.2 .3 Legendre level sets . . . . . . . . . . . . . . . . . . . 14

2.2 .4 Discussion . . . . . . . . . . . . . . . . 15

2.3 Cell splitting methods . . . . . . . . . . . . . . . 17

2.4 Voronoï diagrams . . . . . . . . . . . . . . . . . . . . 19

2.5 Other integrated methods . . . . . . . . . . . . 20

2.6 Discussion . . . . . . . . . . . . . . . . . . 22

3 Segmentation of Multi-Layered Biofilms 23

vii 
3.1 Algorithm . . . . . . . . . . . . . . . . . 25

3.2 Dataset generation . . . . . . . . . . . . 26

3.3 Algorithm initialization . . . . . . . . . . . . . . . 27

3.4 Curvature-based seed selection . . . . . . . . . . . . . 28

3.5 Iterative level set evolution . . . . . . . . . . . . . 28

3.6 Localization of individual bacteria . . . . . . . . . . . . 30

3.7 Stopping criterion and re-initialization . . . . . . . . . . . 31

4 Experimental Results and Analysis $\quad 33$

4.1 Dataset and parameter set up . . . . . . . . . . . . . . 33

4.2 Experimental results . . . . . . . . . . . . . . . 36

4.3 Comparison of segmentation performance $\ldots \ldots \ldots 37$

4.4 Algorithm complexity analysis . . . . . . . . . . . . 41

4.5 Operating time evaluation . . . . . . . . . . . . . . . . 42

4.6 Discussion . . . . . . . . . . . . . . . . . . . 43

5 Improvement and Future Work $\quad 47$

5.1 From active contour to active surface . . . . . . . . . . . 47

5.1.1 Distance-based seed selection . . . . . . . . . . . 49

5.1 .2 Curvature-based active surface model . . . . . . . . 50

5.1.3 Preliminary results and comparison . . . . . . . . . . 51

5.1 .4 discussion . . . . . . . . . . . . . . 53

5.2 Future work: from level set to "lsnake" . . . . . . . . . . . . . 54

6 Conclusion $\quad 55$

$\begin{array}{ll}\text { Bibliography } & 57\end{array}$ 


\section{LIST OF TABLES}

TABLE

Page

4.1 Multi-layered biofilms datasets. . . . . . . . . . . . . . . 34

4.2 Parameter settings for BACT-3D on multi-layered datasets. . . . . . 34

4.3 Comparison of segmentation efficiency (1) . . . . . . . . . . . 39

4.4 Comparison of segmentation efficiency (2) . . . . . . . . . . . . 39

4.5 Operating time of Bact-3D . . . . . . . . . . . . . . . . . . . 42 



\section{List OF Figures}

FIGURE

Page

1.1 Microbial fuel cell technology. . . . . . . . . . . . . . . 2

1.2 Bacterial morphology and biofilms in space. . . . . . . . . . . 4

$1.33 \mathrm{D}$ reconstruction of the bacterial biofilm . . . . . . . . . . . 5

1.4 Microscopy imaging techniques. . . . . . . . . . . . . 5 5

2.1 Typical parametric active contour approaches. . . . . . . . . . . 11

2.2 An example of Level set segmentation. . . . . . . . . . . . . . . . . 12

2.3 Level set segmentation methods comparison on variant intensity data . . . . . . . . . . . . . . . . . 16

2.4 Level set segmentation methods comparison on dense data. . . . . 16

2.5 Contour segmentation. . . . . . . . . . . . . . . . 18

2.6 Cell splitting methods. . . . . . . . . . . . . . . 18

2.7 Voronoï diagram process. . . . . . . . . . . . . . . . . . 19

2.8 Limitation of Voronoï tessellation. . . . . . . . . . . . . . . 20

2.9 Segmentation of time-lapse bacteria. . . . . . . . . . . . . 21

2.10 Single cell tracking of bacteria . . . . . . . . . . . . . . . 22

3.1 Flow chart of Bact-3D Algorithm. . . . . . . . . . . . . . . . . . 24

3.2 Automated layer detection examples. . . . . . . . . . . . . . 27

3.3 Seed selection. . . . . . . . . . . . . . . . . . 28

3.4 Seed selection and level set evolution. . . . . . . . . . . . . . 29

3.5 Example of identification of individual bacteria. . . . . . . . . . . 30

3.6 Set background stopping criterion and its reinitialization. . . . . . . 31 
4.1 Sliced results of multi-layered biofilms. . . . . . . . . . . . 35

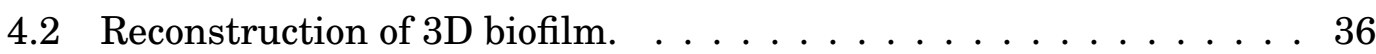

4.3 Results of reconstructed 3D multi-layered biofilms. . . . . . . . . . . 37

4.4 Comparison of segmentation algorithms. . . . . . . . . . . . . 40

4.5 A failure layer detection situation of Bact-3D. . . . . . . . . . . . . 44

4.6 Example of the limitations of Bact3D (simulated data). . . . . . . . 44

4.7 Example of the limitations of Bact3D (real data) . . . . . . . . . . 45

5.1 Demonstration of core innovation designs in improved algorithm: Bact-3Ds. . . . . . . . . . . . . . . . . . . 4 48

5.2 Distance-based seed selection. . . . . . . . . . . . . . . . . 49

5.3 Velocity design of curvature-based active surface model. . . . . . . . 50

5.4 Preliminary results of Bact-3Ds. . . . . . . . . . . . . . 51

5.5 Comparison of improved algorithm with Bact-3D and Chan-Vese in 3D viewpoints. . . . . . . . . . . . . . . . . . 52

5.6 Sliced comparison of improved algorithm with Chan-Vese and Bact3D . . . . . . . . . . . . . . . . . . . . . 53 


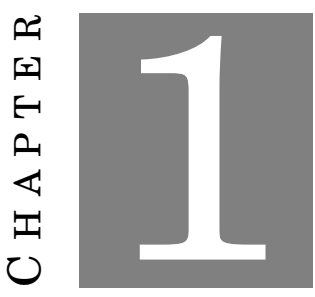

\section{INTRODUCTION}

$\mathrm{s}$ vital participants in natural ecosystems, more than $5 \times 10^{30}[1]$ bacteria inhabit every corner on Earth: soil, water, etc. The majority of them are living in dense aggregations, known as biofilms. This multicellular lifestyle benefits the bacteria by protecting them from their antagonists. For instance, the antibiotic resistance gained by aggregating in those biofilms was found to be 500 to 1000 times of single bacterium [2, 3]. Meanwhile, continuous cell-to-cell cooperations, cell divisions and cell movements happen all the time among multiple species inside these numerous biofilms.

Considering the large quantity of bacteria and biofilms, it becomes significant to explore the relationship between such structures and our daily life. These studies are not only beneficial to decrease the biological risks for human life, but might also proof useful to understand and develop a way to harvest the energy generated from the bacteria. In medicine, researchers found that, it was necessary to administer higher dosage of drugs to treat chronic bacterial infections or the infections caused by medical injection [4]. In the industrial field, the combination of waste water and electrons could produce $\mathrm{CO}_{2}$, protons 
and electrons as well as electrical power as bacteria grows on the conductive graphite fibre shown in Fig. 1.1, which is known as microbial fuel cell technology [5]. It is noteworthy that about $17 \mathrm{GW}$ electrical power could be recovered from industrial and animal wastewater using this technology, which means the technology could effectively make up the consumption of energy for the treatment of organic-rich wastewater in the United States [6].

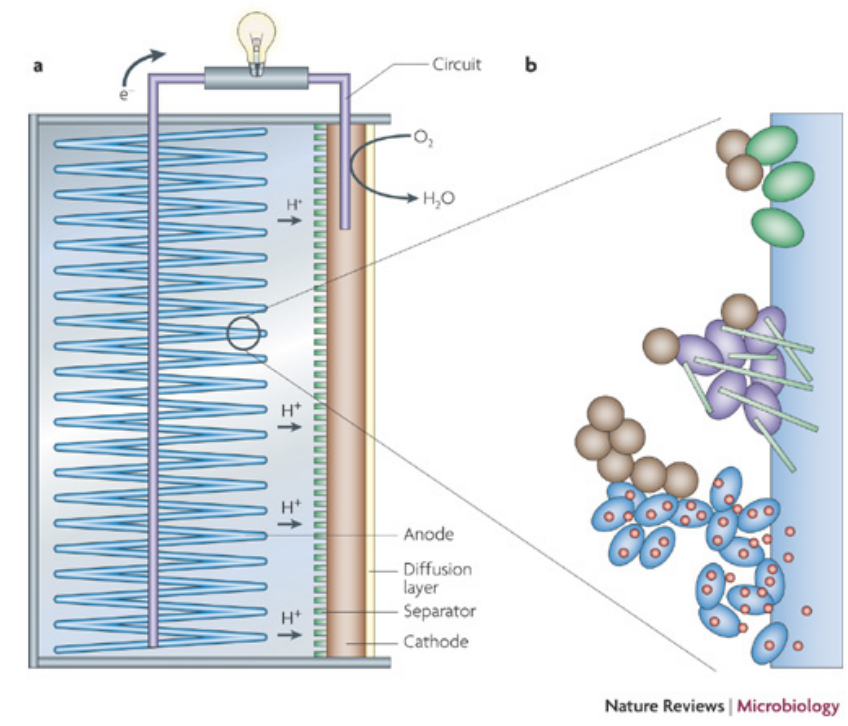

FiguRE 1.1. Microbial fuel cell (MFC) technology. (a) shows the schematic of the whole MFC system; and (b) shows how different types of microorganisms (brown are bacteria) in an anodic biofilm interact with MFC device. Figure from [5].

The bacterial impacts on humans cannot be ignored, but our knowledge of bacteria, especially of the architectural development of bacterial biofilms, has been limited over the past years. For example, many species of bacteria are still unknown and many related diseases are still incurable. Identifying individual bacterium with biofilms and analyzing their behavior becomes crucial in the field of energy, medicine and chemistry. To achieve this, there are two critical steps required in the biofilm analysis: one is a suitable imaging technique for cells of interest (section 1.2); the other is an accurate segmentation technique 
to identify regions of interest (bacteria) within these films (section 1.3 and chapter 2).

\subsection{Bacteria and biofilm basics}

Bacteria, such as coccus, bacilli, vibrio and spirilla, have large diversity in shape. We will primarily focus on bacilli species, in particular Shewanella, which has an ellipsoidal structure in space. In size, this kind of bacteria is about 0.5 to 5.0 micrometers in length and 0.5 to 1 micrometers in diameter. Example of these bacteria is shown in Fig. 1.2.

Bacteria closely gather as a large biofilms, where individual cell division, information exchange, intra- and inter-species cooperation or competition occur all the time. Here, a bacterial "social network" among the cells [7] is generated with extra-cellular membrane protrusions that interconnect cells, which are also part of biofilms (shown in Fig. 1.2).

Take a closer look at the cellular structure of single bacterium (right figure in Fig. 1.2). Each bacterium is composed of outer layers and internal materials. fimbriae, S-layers (cell wall), glycocalyx and flagella are four outer layers. Two of these structures contribute to the formation of biofilms: fimbriae and glycocalyx [8]. Fimbriae is just the structure that helps the bacterium (and other free-floating microorganisms) to attach to a surface (to form a biofilm) or to other cells (to develop communication). Glycocalyx is the structure protecting the materials inside bacterium, which also participates the attachment of bacteria to surfaces to form biofilms. The internal components are "information and nutrient storage": DNA, ribosomes, membranes and cytoskeletons. It is hypothesized in the Gahlmann lab that the activities of ribosomes, the most abundant intracellular multiprotein structures, help improve the electron conductivity of biofilms. To prove this hypothesis, it is also significant to visualize and track these proteins.

To better understand biofilm growth and take advantage of cellular activities, it becomes necessary to observe the behavior of a single bacterium in 

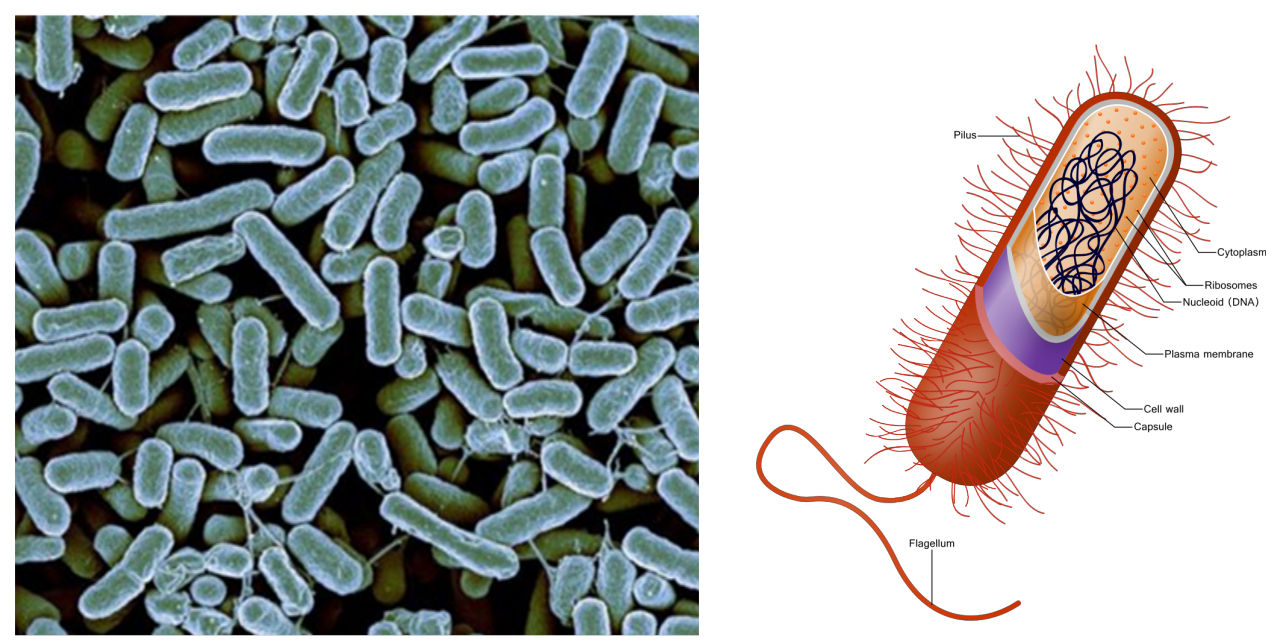

FIGURE 1.2. Bacterial morphology and biofilms in space. Figure on the left is from BBC news [9], figure on the right is from [10].

crowded environments, where one feasible start is imaging of raw bacteria.

\subsection{Imaging techniques}

Over the years, many imaging techniques were developed to visualize and detect the morphology and behavior of bacterial biofilms and intracellular activities [11], such as fluorescent protein tag, immunofluorescence, widefield epifluorescence, structured illumination microscopy, etc. The diffractionlimited resolution of these optical microscopy techniques is the key limitation to observe bacterial activities. Traditional optical confocal microscopy blurs the individual cells together, when biofilm extends in all the directions in $3 \mathrm{D}$ space that beyond the single-cell size. In this case, it is not applicable to localize individual cells (Fig. 1.3). In addition, it was reported in [12] that high light intensities generated during the imaging process damage the bacterial structure, hindering the study of the growth of live bacterial biofilms over time. 


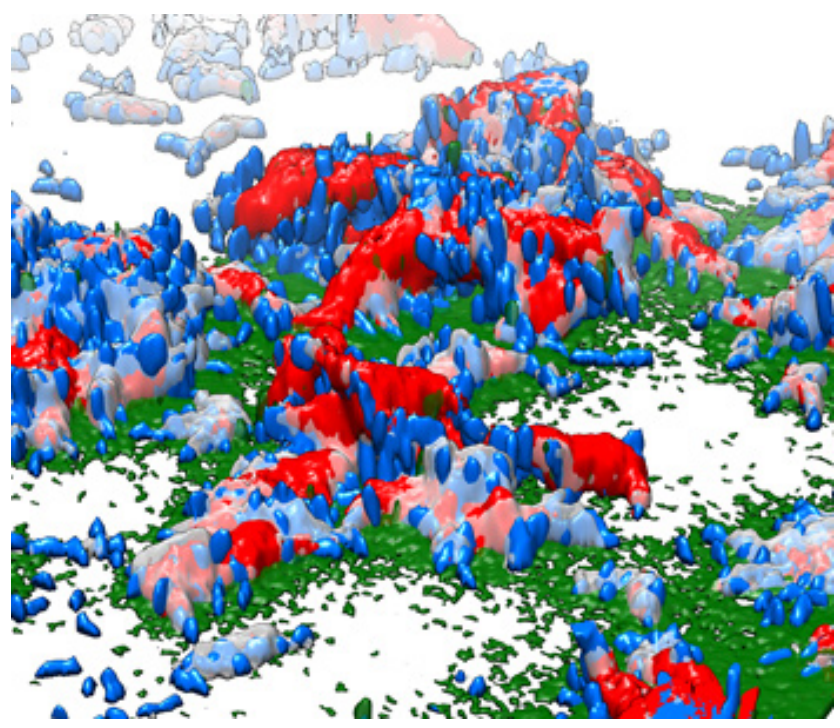

FIGURE 1.3. 3D reconstruction of the bacterial biofilm made by cholera bacteria. Bacteria (blue) are strongly overlap with each other if cells are located deep within a dense 3D biofilm. Figure from [13].
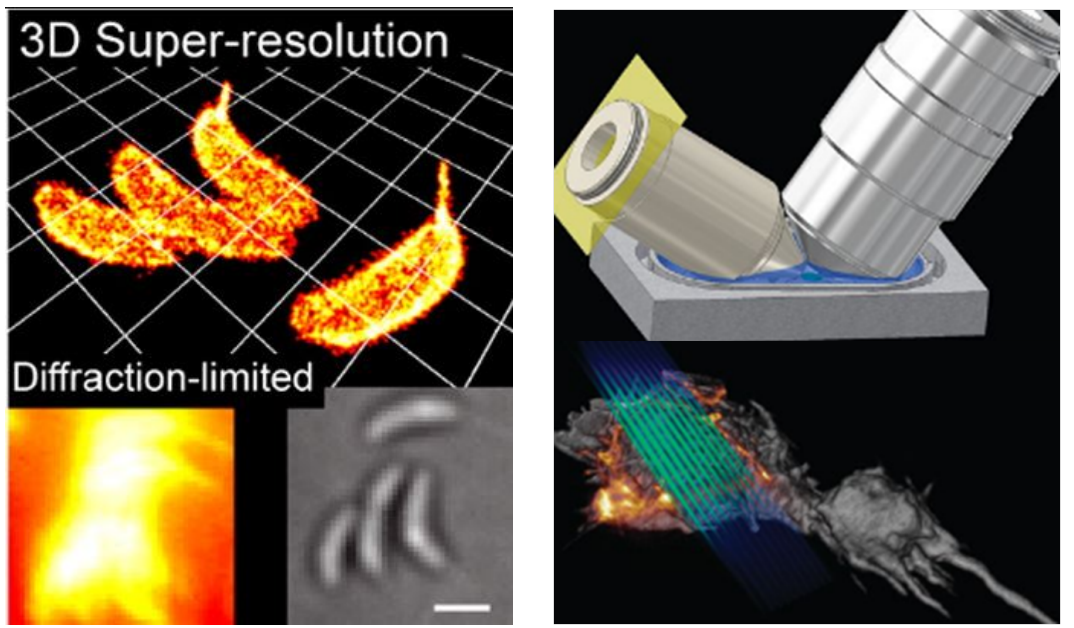

FIGURE 1.4. Microscopy imaging techniques. Left is the comparison of traditional confocal microscopy and super-resolution microscopy. Figure from [14]. Right picture shows how lattice light-sheet microscopy works. Figure from [15]. A structured light sheet (blue-green) scans the specimen (gray) and excites fluorescence (orange) that create the $3 \mathrm{D}$ super-resolution images like the upper left ones. 
Because of the aforementioned limitations, a non-invasive imaging technique is needed. In this thesis, we are exploring the structure of biofilm using $3 \mathrm{D}$ super-resolution imaging technology $[15,16]$, which relies on computational recovery of optical signals. All the live-cell super-resolution images are generated using lattice light-sheet microscopy [15] in the Gahlmann lab in the Department of Chemistry, University of Virginia. This new super-resolution method can work with non-phototoxic illumination intensities as well as work in a traditional diffraction-limited light-sheet microscope mode. It can not only produce three-dimensional images at resolutions two times smaller than that provided by traditional light microscopy, but also avoid the phototoxicity by exciting fluorescence of a specimen in continuous time domain planes via a structured light sheet [15]. Fig. 1.4 shows a comparison of images produced by traditional confocal microscopy and by super-resolution microscopy.

\subsection{Segmentation techniques overview}

Being a technique that partitions an image into multiple regions of interest (ROI), segmentation [17] offers a way to study the architecture of bacterial biofilm and behavior of individual bacterium inside those complex biofilms after biomedical imaging.

In general, the segmentation techniques can be classified to mainly four types [18]:

(1) Pixel-based segmentation, such as thresholding, which is the simplest way that extract the pixel-level image features (e.g. gray-level values, histogram[19]);

(2) Edge-based segmentation relies on the position of edges, where gradient and Hessian of the image give a notable signal to define the contour. This kind of technique detects the discontinuities in color, texture, etc. [20], and applies further processing to present smooth edges, for instance edge detection [21];

(3) Region-based segmentation analyzes the relationship of connected 
pixels and then classifies isolated areas to homogeneous subsets of the image. This gives us information about individual objects. Some main techniques could be region growing [22] covering regions with similar neighboring pixels, watershed [23], etc.;

(4) Model-based segmentation has the ability to identify different objects globally, which means it gathers the information from all the local operators and uses the pre-knowledge of target object to pick out ROI's in a data set, such as deformable models [24].

However, these classic segmentation method are not comprehensive. On top of that, they have many limitations: for example, edge-based segmentation is heavily affected by noise and region-based segmentation is hard to find a criteria for identifying similarity. As a result, dynamic solutions (level set [25], snake [26], graph cut [27], etc.) combines multiple segmentation types, are becoming more popular for complicated datasets. In this thesis, typical segmentation techniques, especially for cellular microscopy segmentation, will be discussed in chapter 2 and later.

\subsection{Thesis overview}

This thesis presents a procedure of identifying the morphology of dense bacterial bioflims with proteins labeled in each bacterium using super-resolution imaging technique. Bact-3D is proposed in this thesis which detects and tracks position and orientation changes with depth, from a densely packed multilayered 3D volume. We exploit the idea of over-segmentation [28] to identify the location and orientation of the bacteria within each slice in the $z$-direction, while simultaneously propagating the background (non-bacteria) detection to the subsequent slices to retain the separability between individual bacteria. The different bacterial layers within the biofilm are identified by detecting transitions in the number of detected cells as function of slice location along the $z$-direction.

In Chapter 2 of the thesis, more previous bacterial segmentation methods 
will be discussed and implemented. In Chapter 3, the algorithms demonstrated for the research are presented and explained followed by the implementation of the overall method and discussion comparing with other methods in Chapter 4. In Chapter 5, ideas to improve and enhance the robustness of Bact-3D on random distributed datasets are proposed. Finally, the thesis will end up with a conclusion in Chapter 6. 


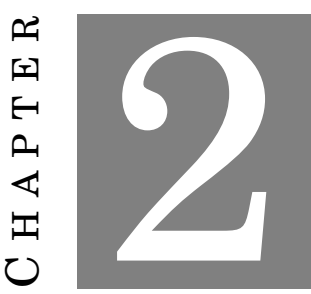

\section{SEGMENTATION BACKGROUND}

utomatic segmentation on biomedical cell images is always difficult because of various challenges [29] (diversity in imaging types, density of cells, different cell structures, noise influences, etc.). Nevertheless, numerous segmentation approaches, such as [17, 23, 25, 30-33], are proposed to segment those varied biomedical microscopy images over the past few years. In this chapter, some of the most relevant and inspiring approaches are highlighted, investigated and evaluated.

\subsection{Parametric active contours}

The emergence of parametric active contour, snakes, could be traced back to about 30 years ago when Kass, et al. [26], proposed their first generation of parameterized curve to detect object boundaries. Generally, the snakes (parametric active contours) deform on the image domain and capture the desired feature by minimizing an energy functional (Eq. 2.1) subject to certain constraints (Eq.):

$$
E_{\text {snake }}=E_{\text {internal }}(\mathbf{v}(s))+E_{\text {external }}(\mathbf{v}(s))
$$


In this functional, a parameterized continuous curve $\mathbf{v}(s)$ is defined by $\mathbf{x}$ and y coordinates on a $2 \mathrm{D}$ plane. Both $x$ and $y$ coordinate values, represented by $[x(s), y(s)]$, are continuous functions of a scalar parameter $s \in[0,1]$. In this case, $\mathbf{v}(0)=[x(0), y(0)]$ and $\mathbf{v}(1)=[x(1), y(1)]$ are the two ends of the contour, so a closed curve is simply represented by $\mathbf{v}(0)=\mathbf{v}(1)$. This curve is commonly initialized according to the shape and position of targeted ROIs manually and then evolves to the optimized position by finding the least-energy solution of Eq. 2.1. In particular, some methods could also provide automated initializations, for example the Poisson inverse gradient (PIG) method[34].

Two forces contribute to the evolution of the energy functional, $E_{\text {internal }}$ and $E_{\text {external }}$. The internal force defined in [26], shown in Eq. 2.2, makes the curve smooth and taut, where $\alpha, \beta$ are just tunning parameters:

$$
\begin{aligned}
E_{\text {internal }}(\mathbf{v}(s)) & =\int_{0}^{1} \frac{1}{2}\left(\alpha\left|\mathbf{v}^{\prime}(s)\right|^{2}+\beta\left|\mathbf{v}^{\prime \prime}(s)\right|^{2}\right) \\
& =\frac{1}{2} \int_{0}^{1} \alpha\left(\left|\frac{\mathrm{d} x}{\mathrm{~d} s}\right|^{2}+\left|\frac{\mathrm{d} y}{\mathrm{~d} s}\right|^{2}\right)+\beta\left(\left|\frac{\mathrm{d}^{2} x}{\mathrm{~d} s^{2}}\right|^{2}+\left|\frac{\mathrm{d}^{2} y}{\mathrm{~d} s^{2}}\right|^{2}\right)
\end{aligned}
$$

Another force, external force, which is chosen to be the potential field developed by image gradient magnitude in [26], attracts the elastic model to ROIs. It is a flexible term with no fixed definitions or expressions, which makes external force become one key term to define snakes to specific situations.

Typical external forces that are widely used in biomedical image segmentation, are gradient vector flow (GVF) that establish a force field from the gradient of the image [32], and vector field convolution (VFC) [35] which defines a vector field kernel to convolve with the edge map. A quick visualization of how these two methods work shown in Fig. 2.1 [35].

There are two limitations related to parametric snakes. One is the lack of flexibility of curve initialization. In a random-distributed dense data plane, it is hard to localize all the ROI's and set the original curve around these ROI's. Another limitation is the calculation cost for a $3 \mathrm{D}$ parametric active contour. On one hand it needs much more parameters to define a deformable surface; 
on the other hand, it is more complicated to manually define a force field in space.

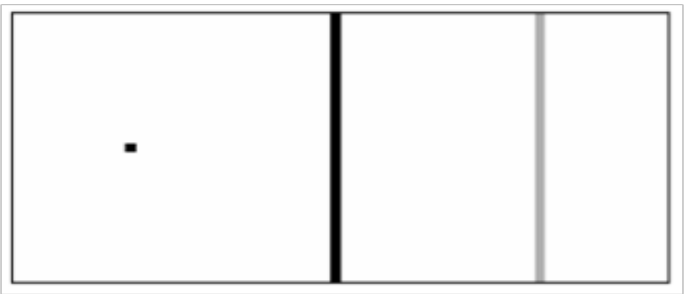

(a) Edge map

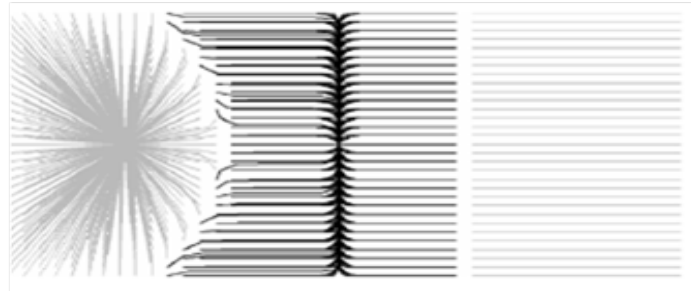

(b) GVF

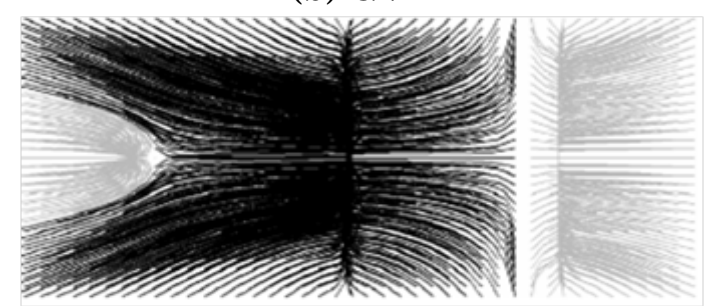

(c) VFC

FIGURE 2.1. Typical parametric active contour approaches. (a) includes an impulse noise, a strong edge and a weak edge, where noise and strong edge have the same magnitude that is four times of the weak one. (b) and (c) are force field, using GVF [32] and VFC [35], generated from (a). In this comparison, VFC can not only work on weak edges but also diminish the influence of noise, which means it shows a better performance to attract free particles to edges. Figures from [35] 


\subsection{Geometric active contours}

Geometric active contours (level set methods), which were initially proposed by Osher and Sethian [36] in 1988, presented an efficient approach for segmenting multiple objects at the same time automatically. A prominent advantage is that the segmentation results do not rely on the curve initialization, which proves a better performance on the random-distributed data than aforementioned parametric active contour segmentation techniques.

\subsubsection{General expression}

Mathematically, in the level set segmentation approach proposed in [36], a signed Euclidean distance function $\phi(x, y ; t)$ iteratively updates the distances to the closest boundary positions at each iteration $t$. The object boundary is defined by the zero level set. Fig. 2.2 gives an example of the level set approach.

$$
C=\{(x, y ; t): \phi(x, y ; t)=0\}
$$

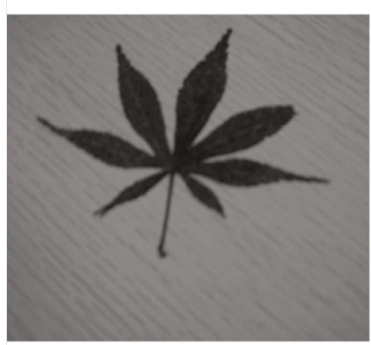

(a)

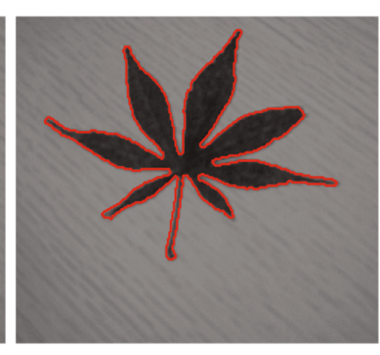

(b)

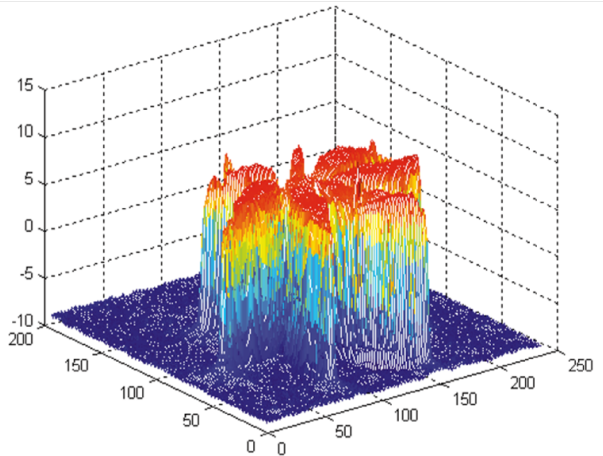

(c)

FigURE 2.2. An example of Level set segmentation. Figures from [35] 
The inside of the contour is denoted by $\phi>0$, while the outside is $\phi<0$ (this will vary in different cases). The motion of the level set could be expressed as the first derivative respecting to time $t$ :

$$
\frac{\partial C}{\partial \mathrm{t}}=\mathrm{VN}
$$

where $\mathrm{V}$ is the velocity of the evolving curve and $\mathbf{N}=\nabla \phi /|\nabla \phi|$ is the outward normal force. The curve moves in this normal force direction with specific design of velocity $\mathrm{V}$ and other constraints to stop the evolution. Using the chain rule, the general expression for the evolution of $\phi$ and hence the contour is derived as follows where the partial derivative of $\phi$ is set to be zero to get a steady state of contour:

$$
\begin{aligned}
0=\frac{\partial \phi(x, y ; t)}{\partial \mathrm{t}} & =\phi_{x} x_{t}+\phi_{y} y_{t}+\phi_{t} \\
\Longrightarrow \phi_{t} & =-\left(\phi_{x} x_{t}+\phi_{y} y_{t}\right) \\
& =-<\nabla \phi, C_{t}>=-<\nabla \phi, \mathrm{VN}> \\
& =-V<\nabla \phi, \nabla \phi /|\nabla \phi|>=-\mathrm{V}|\nabla \phi|
\end{aligned}
$$

In this formula, velocity $\mathrm{V}$ can be designed in different expressions to deal with different datasets. Following are some examples.

$$
\begin{gathered}
\text { Constant flow : } C_{t}=\mathbf{N} \Longleftrightarrow \phi_{t}=|\nabla \phi| \\
\text { Curvature flow : } C_{t}=\kappa \mathbf{N} \Longleftrightarrow \phi_{t}=\operatorname{div}\left(\frac{\nabla \phi}{|\nabla \phi|}\right)|\nabla \phi|
\end{gathered}
$$

Kass, et al. [36] : $C_{t}=(g \kappa-<\nabla g, \mathbf{N}>) \mathbf{N} \Longleftrightarrow \phi_{t}=\operatorname{div}\left(g \frac{\nabla \phi}{|\nabla \phi|}\right)|\nabla \phi|$

where $\mathrm{g}$ is the edge indicator function:

$$
g=\frac{1}{1+\left|\nabla\left(G_{\sigma} \times I\right)\right|^{2}}
$$

and $\kappa$ is level set curvature:

$$
\kappa=\operatorname{div}\left(\frac{\nabla \phi}{|\nabla \phi|}\right)
$$

In above, evolution of Eq. 2.8 depends on the edge of the object by indicator function Eq. 2.9, which requires a clear boundary to attract and stop the level set; otherwise, the level set could easily "leak" through the object boundary. 


\subsubsection{Active contours without edges}

To overcome the leakage problem, a classic method geometric active contour without edges was proposed by Chan and Vese [25] in 2001. In this paper, the images are modeled into two regions: foreground and background. The zero level set is found by computing the optimal solution of the energy functional:

$$
\begin{aligned}
\varepsilon\left(\phi, \mu_{\text {fore }}, \mu_{\text {back }}\right) & =\lambda_{1} \int_{\Omega}\left(I-\mu_{\text {fore }}\right)^{2} H(\phi) d \Omega \\
& +\lambda_{2} \int_{\Omega}\left(I-\mu_{\text {back }}\right)^{2}(1-H(\phi)) d \Omega \\
& +\alpha \int_{\Omega} H(\phi) d \Omega+\beta \int_{\Omega}|\nabla H(\phi)| d \Omega
\end{aligned}
$$

Correspondingly, the evolution partial derivative equation in the level set is:

$$
\phi_{t}=\delta(\phi)\left[\lambda_{2}\left(I-\mu_{b a c k}\right)^{2}-\lambda_{1}\left(I-\mu_{\text {fore }}\right)^{2}-\alpha+\beta \operatorname{div}\left(\frac{\nabla \phi}{|\nabla \phi|}\right)\right]
$$

In these two equations, $\delta(\phi)$ is the impulse function that equals $\frac{\mathrm{d} H(\phi)}{\mathrm{d} \phi} \cdot \mu_{\text {fore }}, \mu_{\text {back }}$ denote the mean intensity values inside and outside the zero level set. $\lambda_{1}, \lambda_{2}, \alpha, \beta$ are regularization parameters. $H(\phi)$ is the Heaviside function:

$$
H(x)=\frac{1}{2}\left(1+\frac{2}{\pi} \tan ^{-1}\left(\frac{x}{\epsilon}\right)\right)
$$

which indicates the area $\left(\int_{\Omega} H(\phi) d \Omega\right)$ and the length $\left(\int_{\Omega}|\nabla H(\phi)| d \Omega\right)$ of the zero level set.

This level-set based segmentation model [25] can efficiently separate homogeneous intensity region, but fails to capture the inhomogeneity inside objects to segment. Examples are shown in Fig. 2.3 (b).

\subsubsection{Legendre level sets}

Recently, the authors in [30], proposed a modified Chan-Vese technique where intensity variations within imaged regions are modeled as linear combinations of Legendre polynomial functions. In this paper, Eq. 2.11 was 
rewritten as:

$$
\begin{aligned}
\varepsilon^{m}(\phi, A, B) & =\int_{\Omega}\left|f(\mathbf{x})-A^{T} \mathbb{P}(\mathbf{x})\right|^{2} H(\phi) d \mathbf{x}+\lambda_{1}\|A\|_{2}^{2} \\
& +\int_{\Omega}\left|f(\mathbf{x})-B^{T} \mathbb{P}(\mathbf{x})\right|^{2}(1-H(\phi)) d \mathbf{x}+\lambda_{2}\|B\|_{2}^{2} \\
& +v \int_{\Omega} \delta(\phi) \frac{\nabla \phi}{|\nabla \phi|} d \mathbf{x}
\end{aligned}
$$

where $\mathbb{P}(\mathbf{x})$ denotes the vector of Legendre polynomials with $A$ and $B$ representing coefficient vectors for the two regions. The Heaviside function $H(\phi)$ is used to identify the regions inside of the curve $(H(\phi))$ and the regions outside of the curve $(1-H(\phi)) . \lambda_{1}$ and $\lambda_{2}$ in front of the regularization terms (denoted by $\|\bullet\|_{2}^{2}$ ) are used to avoid over-fitting. $v$ is just a tuning parameter to smooth the contour. In this case, the evolution of Legendre level set (L2S) model is perform by solving:

$$
\phi_{t}=\delta(\phi)\left[-\left|f(\mathbf{x})-\hat{A}^{T} \mathbb{P}(\mathbf{x})\right|^{2}+\left|f(\mathbf{x})-\hat{B}^{T} \mathbb{P}(\mathbf{x})\right|^{2}+v \operatorname{div}\left(\frac{\nabla \phi}{|\nabla \phi|}\right)\right]
$$

The regions of foreground and background are represented by computing coefficient vectors: $A^{T} \mathbb{P}$ and $B^{T} \mathbb{P}$. These two closed-formed vectors not only make the evolution faster and more efficient than previous methods, but also have the ability to compute coefficient vectors representing variant intensities automatically. In another word, L2S exhibits better performance on intensity inhomogeneous images than Chan-Vese does. The comparison of these two methods is shown in Fig. 2.3.

\subsubsection{Discussion}

Level set segmentation techniques present an alternative way to deal with biomedical images, with both uniformly distributed intensities and those with variant intensities. These methods are efficient in extracting regions of interest from images, but they are often not able to identify multiple densely packed objects or regions. For example, the image shown in Fig. 2.4 (a) is one slice of dense bacteria biofilm simulated data using super-resolution imaging technique. L2S [30] shows a quick and accurate result to detect the contour 
of the dense data; however, the cells are not separated individually which becomes a problem for tracking and observing individual bacterium behaviors.
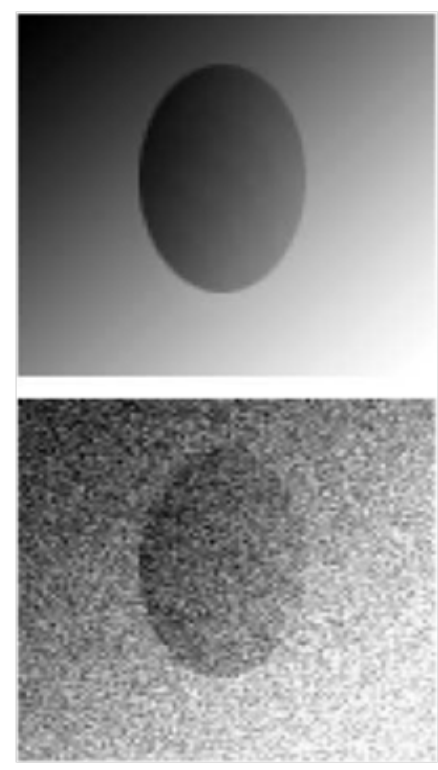

(a)
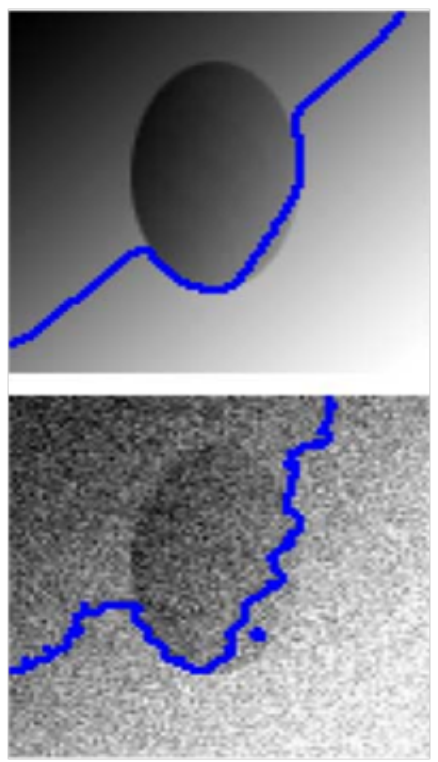

(b)
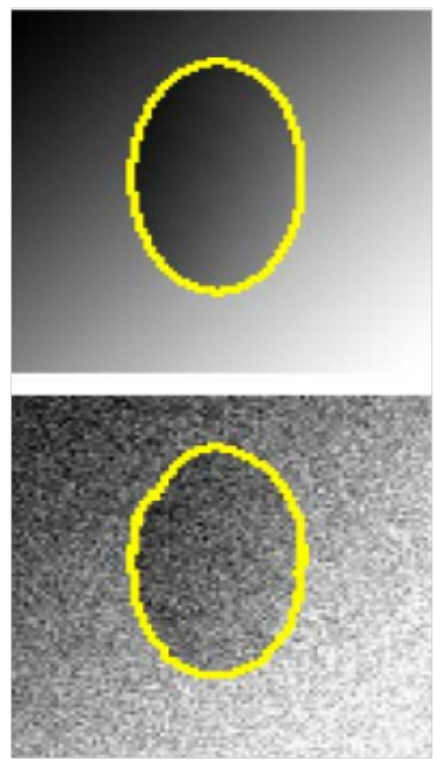

(c)

FIGURE 2.3. Level set segmentation methods comparison on variant intensity data between Chan-Vese [25] (b) and L2S [30] (c). The second row of original image (a) adds a Gaussian noise. Figures captured from [30]

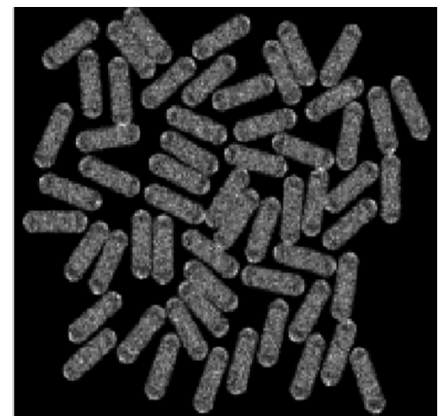

(a)

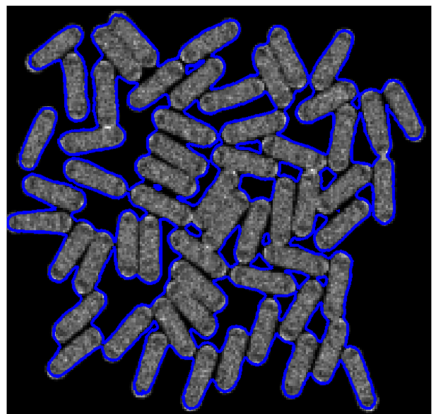

(b)

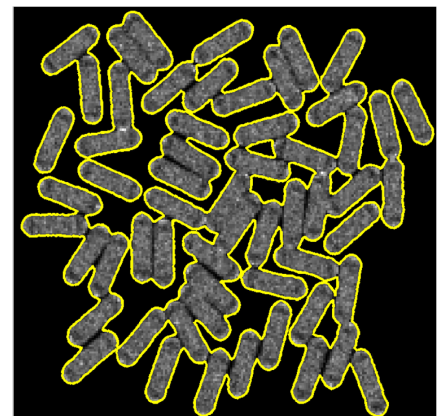

(c)

FIGURE 2.4. Level set segmentation methods comparison on dense data between Chan-Vese [25] (b) and L2S [30] (c). Original image (a) is in the original dataset used in this thesis. 


\subsection{Cell splitting methods}

In order to differentiate the individual regions of interest (bacteria in our case), algorithms which can identify and leverage small separation between regions are desired. For example, [23] uses the gradient flow to identify segment contours. The algorithm will automatically find the closest valleys (steepest descent) along the ridges in the images, where the images are treated as topographic maps (Fig. 2.6 (b), row 1). The process of this method is shown in the first row of Fig. 2.6. For this approach, situations where the intensity of the regions of interest is non-homogeneous often lead to incorrect segmentation (Fig. 2.6 column (c), row 1) .

Another method proposed in [37] attempts to separate large segmented regions by identifying concave points along the contour. The concave points are extracted by calculating and comparing the concavities, where the concavity is defined as the absolute angle difference between previous points $p_{\text {pre }}$ and next points $p_{\text {next }}$ relative to detected current point $p_{c}$ :

$$
\text { Concavity }=\left\{\begin{array}{l}
\left|\angle\left(p_{\text {pre }}, p_{c}\right)-\angle\left(p_{\text {next }}, p_{c}\right)\right|, \text { if } \text { Concavity }<\pi \\
\pi-\left|\angle\left(p_{\text {pre }}, p_{c}\right)-\angle\left(p_{\text {next }}, p_{c}\right)\right|, \quad \text { otherwise }
\end{array}\right.
$$

The authors in [37] picked out the points that satisfy the following criterion to be the concave points:

(1) The concavity is in a limited range, $a_{1}<$ Concavity $<a_{2}$;

(2) The line that connects neighboring detected points does not pass through the cells.

This step helps to divide the contour into several segments ( $L_{1}$ and $L_{2}$ in Fig. 2.5). Ellipse fitting [38] is then applied individually to each segments, reconstructing an approximate cell boundary (Fig. row 2, 2.6 (c)). However, multiple concave points present on the contours (examples shown in the red region marked in Fig. 2.5 (b)) do not necessarily denote the presence of adjacent cells. On top of that, it is hard to decide which two points are supposed to be connected to split joint contours into different cells. 


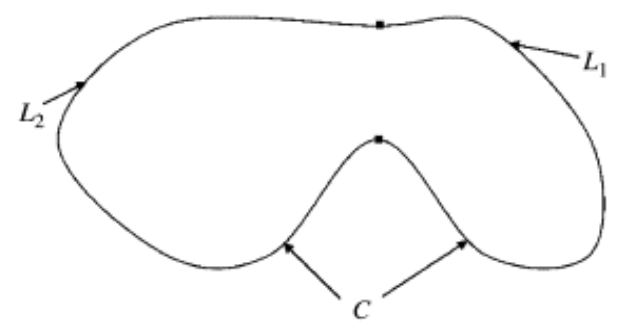

(a)

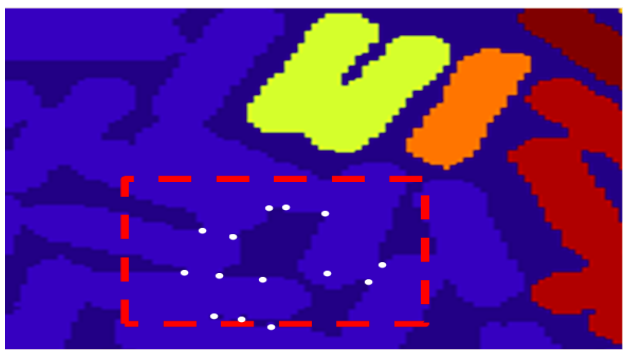

(b)

FIGURE 2.5. Contour segmentation. (a) In theory, contour $\mathrm{C}$ is divided into $L_{1}$ and $L_{2}$. Figure from [37]. However, (b) shows a failure situation to apply [37]. White points are concave points with distinct concavity change along the contour.
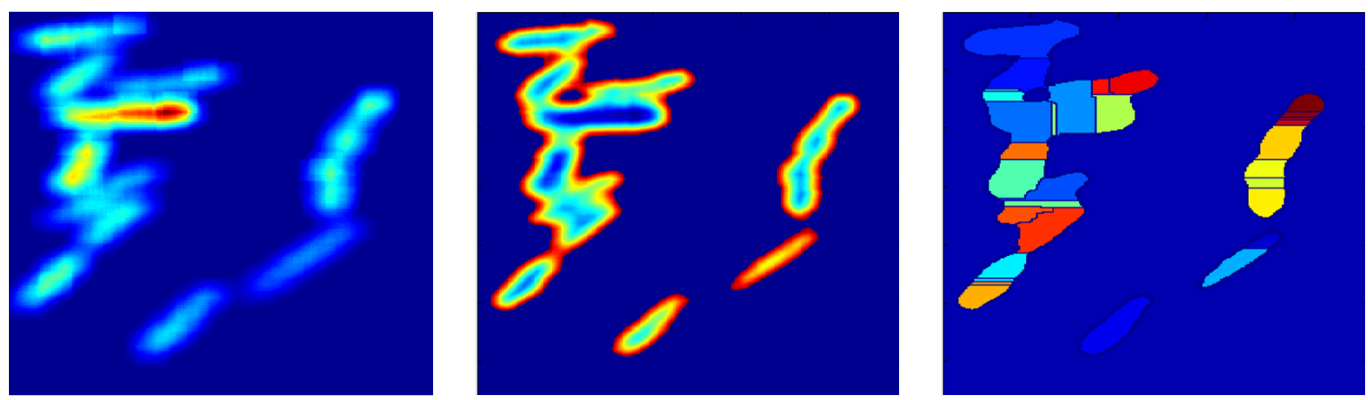

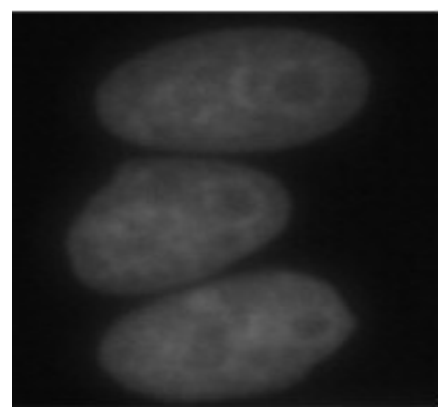

(a)

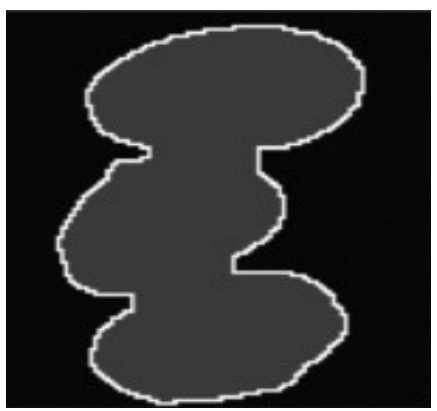

(b)

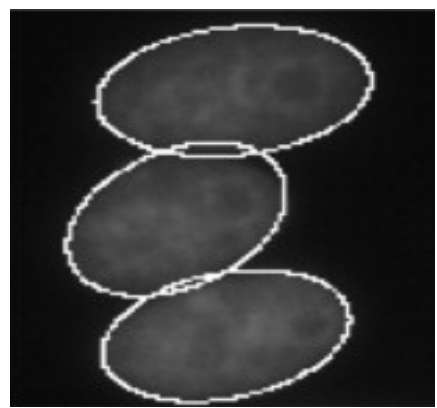

(c)

FIGURE 2.6. Cell splitting methods. Column (a) is the original images. (b) shows the intermediate segmentation results before splitting algorithms. Column (c) shows the final results. Row 1 uses Watershed method [23] (In the first two columns, red means high values while blue means low values). Row 2 shows the results from Bai's cell splitting method [37]. 


\subsection{Voronoï diagrams}

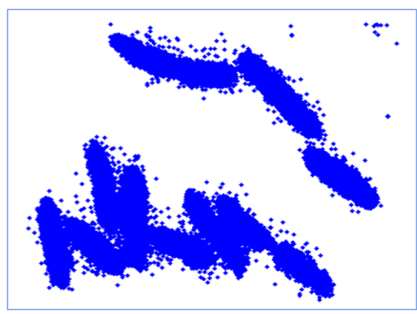

(a)

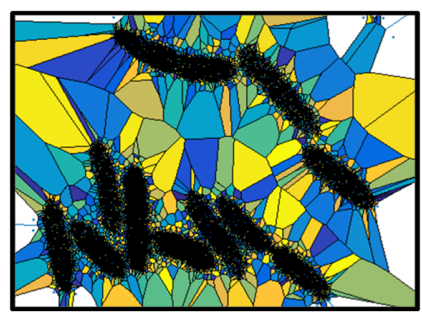

(d)

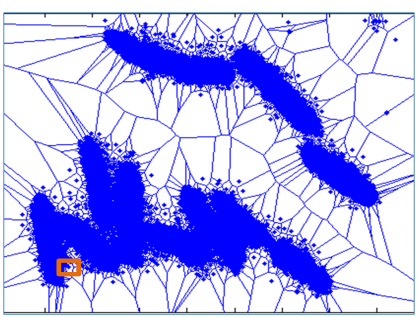

(b)

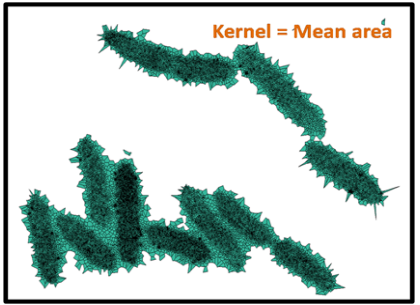

$(\mathrm{e}-1)$

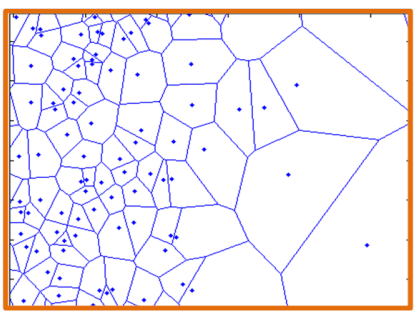

(c)

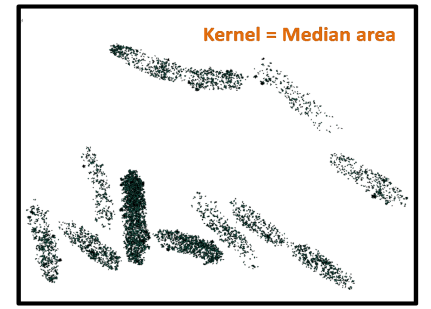

$(\mathrm{e}-2)$

FiguRE 2.7. Voronoï diagram process. (a): the original superresolution image with ribsome marked inside without burring. (b): results after creating voronoï polygons. (c): larger version of the orange region in (b). Patch different polygon areas in (b) give (d). Different thresholding values of polygon areas are tuned in (e) to get the ROI's.

From a different path, Voronoï diagrams (also called Voronoi tessellations) provides an efficient way to segment super-resolution images without Gaussian blurring. As applied in [39], the Voronoï tessellation method partitions an image into several polygons based on the Euclidean distance between two points, where it just allows one point occupy in one polygon. The edges of these polygons are chosen in the equidistant positions between two closest points. This process is shown in Fig. 2.7 (a) to (c). During this process, the area of polygon becomes a breakthrough point to find the ROI's. Because the cell part have higher density of proteins (that is the dots in the image are closer to each other), the corresponding polygons will have smaller areas. We can choose 
these areas using appropriate thresholding value to pick out ROI's as shown in Fig. 2.7 (e).

The limitation of this method is shown in Fig. 2.8 (b). After tuning parameters, it is still unable to separate close cells where the distances between points in the gaps have not much difference with the points inside of one single cell.

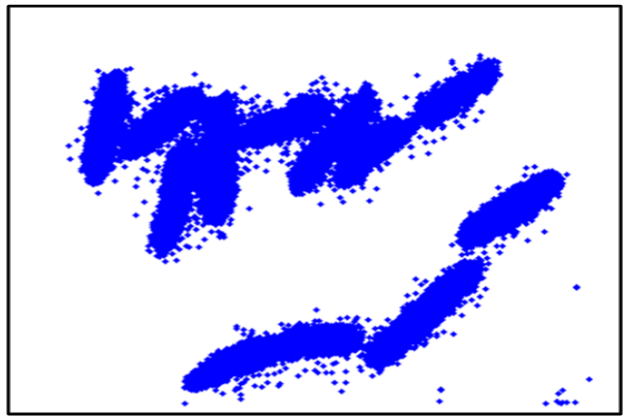

(a)

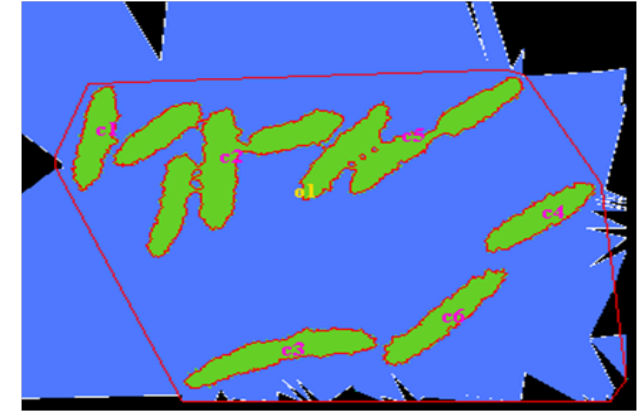

(b)

FIGURE 2.8. Limitation of Voronoï tessellation. (a) is the original super-resolution data marked by points. (b) is the corresponding results using integrated software SR-TESSLER in [39].

\subsection{Other integrated methods}

Besides the existing literatures there are also many applications combining several classic theories to segment biomedical cells. A recent work on the segmentation of time-lapse images of bacteria was proposed in [40] where the authors presented a robust method to track bacteria in dense mono-layer film using an iterative threshold-based approach. In this method, images are enhanced based on bacteria curvature features shown in Fig. 2.9. Then multiple thresholds are selected according to the prior knowledge about cell area and major axis and minor axis length of the cells. During this process, a weight (Eq. 2.17) is assigned to each object in each thresholding level.

$$
\text { weight }=0.5 \times R A R+0.5 \times \text { convexity }
$$


RAR and convexity are defined in Eq. 2.18, where ellipsearea is a given parameter according to prior knowledge.

$$
\begin{aligned}
R A R & =\frac{\min \left(\text { area }_{\text {object }}, \text { ellipsearea }\right)}{\max \left(\text { area }_{\text {object }}, \text { ellipsearea }\right)} \\
\text { convextity } & =\frac{\text { area }_{\text {object }}}{\text { area }_{\text {convexhullofobject }}}
\end{aligned}
$$

However, this method is not applicable to multi-layered bacteria bioflim, such as those we analyze in this work, where the orientation and position of the bacteria change significantly with depth of the biofilm.

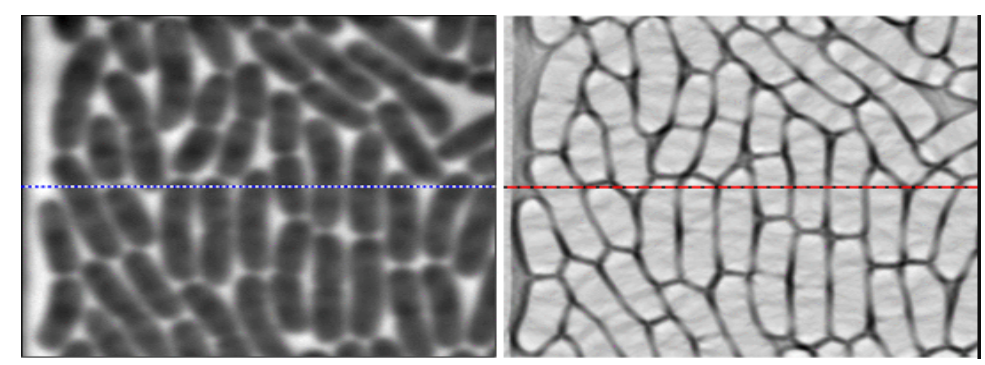

FIGURE 2.9. Segmentation of time-lapse bacteria on curvature-based contrast enhanced image. Left is the original image and right is the contrast enhanced image. Figures are captured from [40].

The segmentation algorithm presented in [41] allows to track bacterial growth in a dense multi-layered community. It captures the projection of raw images by applying watershed [23] on images after deconvolution. This process is shown in Fig. 2.10 A-C. The reconstruction of the community is realized by connecting adjacent voxels to decide the projection orientation and substituting the projections with ellipsoids in 3D (Fig. 2.10 D).

Similarly to previous approaches, this method struggles with the detection of individual bacterial regions for which the intra-region density is not uniform. For example, The image shown in Fig. 2.10 E is not well-segmented using this single cell tracking method with a result in Fig. 2.10 F. One single cell is broken to several parts due to the variant intensity. 

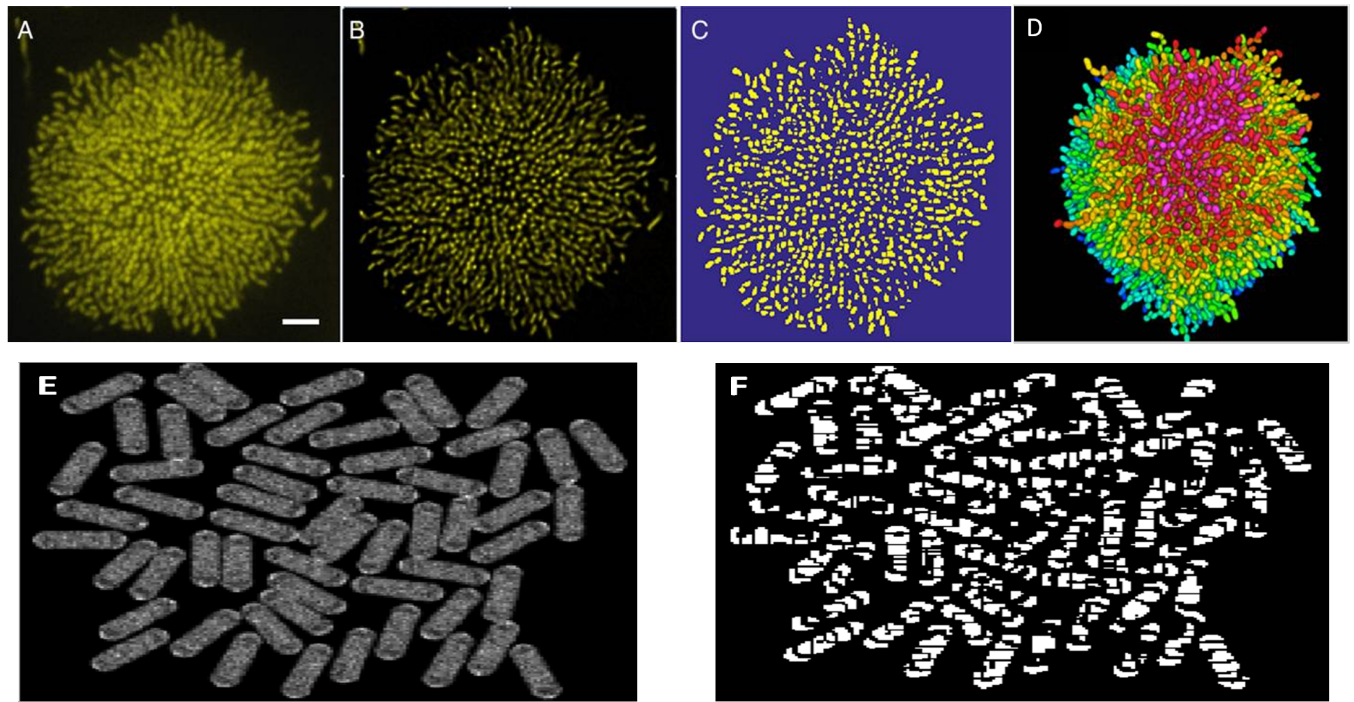

FIGURE 2.10. Single cell tracking of bacteria. A is the raw image. B is the image after deconvolution. $\mathrm{C}$ is after watershed [23]. Figures are captured from [41]. D is a failure situation in our dataset with a segmentation result $\mathrm{F}$.

\subsection{Discussion}

In this chapter, several methods from the literature are reviewed. When applied to our datasets, many limitations are salient, such as segmentation failure on variant intensity of the cells, small separations between closely neighboring cells and difficulty in identifying cell orientations. We are motivated by these limitations and also inspired during the literature review process. Therefore, we put forward our solutions to deal with multi-layered dense dataset in the next chapter. 


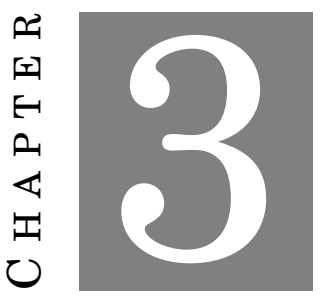

\section{SEgMentation OF MULTi-LAYERED Biofilms}

I

$\mathrm{n}$ order to segment and identify the individual bacteria in 3D dense multilayered biofilms, Bact-3D is proposed in this thesis. It is an approach

which detects and tracks position and orientation changes with depth, from a densely packed multi-layered 3D volume. We exploit the idea of oversegmentation [28] to identify the location and orientation of the bacteria within each slice in the $z$-direction, while simultaneously propagating the background (non-bacteria) detection to the subsequent slices to retain the separability between individual bacteria.

Fig. 3.1 shows a flow diagram of the whole approach. We start at the initial layer slice that is detected by automated layer detection method (sec. 3.3, Fig. 3.1 a). Then, local level set evolution (sec. 3.5) iterates from the seed (Fig. 3.1 b) selected in sec. 3.4, which will end up with a signed distance function (Fig. $3.1 \mathrm{c})$. Morphological clean-up procedures are followed to abandon non-bacteria components which gives preliminary contour marked with different colors (Fig. $3.1 \mathrm{~d}$ ). Identification of every bacterium in later sec. 3.6 uses least square fitting [38]. Finally, a stopping criterion of the smoothed background would be set up and updated to later slices as stated in sec. 3.7. 


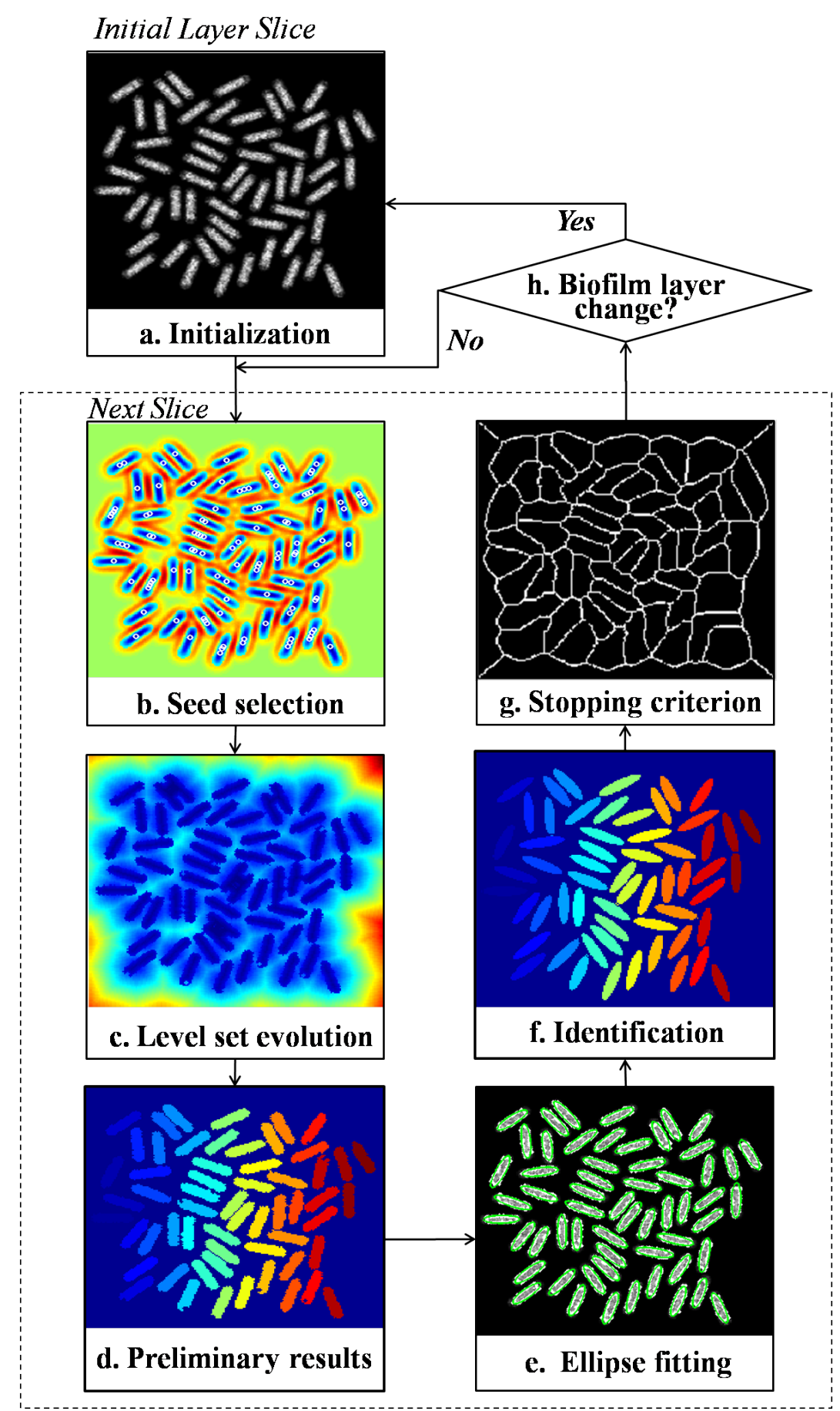

FigURE 3.1. Flow chart of Bact-3D Algorithm. 


\subsection{Algorithm}

The following shows the implementation of this algorithm, where $3 \mathrm{D}$ bacterial biofilms $\left(V_{c}\right)$ are reconstructed by stacking up the $2 \mathrm{D}$ segmentation results along $z$-direction from the initial layer $\left(I_{0}\right)$ detected by the automated layer detection to the end of the volume.

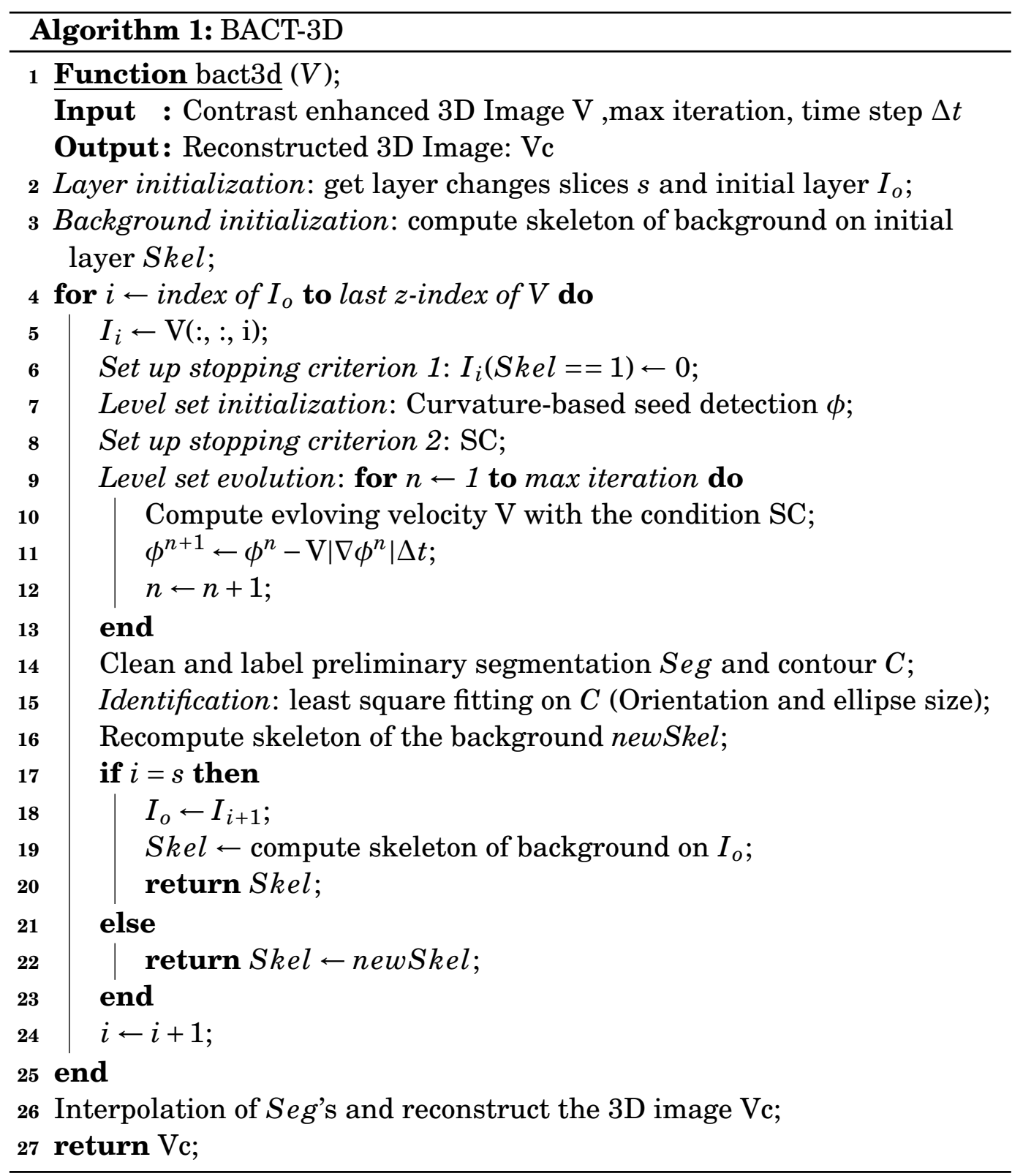


The algorithm integrates functions for layer detection, background stopping criterion initialization, 2D level set evolution, localization of individual bacterium and reconstruction of 3D biofilms, which are introduced in the following sections in this chapter. The implementation of $2 \mathrm{D}$ segmentation in each slices (Algorithm 1, line 4-25) can be divided into three steps. The first step is achieving preliminary segmentation results using iterative local level set method (Algorithm 1, line 7-13). The second step is identifying individual bacterium from line 14 to 15 in Algorithm 1,. Lastly, a decision to reinitialize the stopping criterion is made from line 17 to 23 in Algorithm 1,.

\subsection{Dataset generation}

To evaluate the accuracy of our proposed approach and to compare it to existing methods, we generated five different data sets that closely replicate the fluorescence emission from a tightly-packed cluster of bacteria, similar to those found in a microbial biofilm grown on an agarose pad.

Each data set was generated using a two-step algorithm. First, bacterial cell volumes were approximated as cylinders, $2 \mu \mathrm{m}$ in length and of radius $0.5 \mu \mathrm{m}$, with hemispherical end caps of radius $0.5 \mu \mathrm{m}$. These volumes were randomly filled with 10,000 points drawn from a spatially-uniform distribution. Cells were then randomly rotated in the $x-y$ plane and placed by a packing algorithm within $20 \mu \mathrm{m} \times 20 \mu \mathrm{m}$ field of view. The algorithm shifted each cell based on its proximity to its neighbors until no overlap between adjacent cells occurred. The generated cell layer was then rotated twice in 90 degree increments to create two more cell layers that were positioned above each other and on top of the original layer. The final result closely simulates a three layer structure where each point represents an individual fluorescence emitter. In the second step, the points in each data set were convolved with a Gaussian kernel and then summed to generate light intensity values over a grid of $200 \times 200 \times 100$ voxels, where each voxel represents a cube of $100 \mathrm{~nm}$ mimicking experimental constraints. The size and symmetry of the Gaussian kernel 
were chosen to closely mimic the anisotropic spatial resolution encountered experimentally: $\sigma_{x}=\sigma_{y}=77 \mathrm{~nm}$ and $\sigma_{z}=192 \mathrm{~nm}$.

\subsection{Algorithm initialization}

Although bacteria are densely packed, their ellipsoidal shape, due to the interaction between the distribution of fluorophores and the simulated point spread function, helps in identifying a $z$-plane where all the individual cells are completely separated.

To automatically identify this starting slice we analyze the number of detected bacteria as function of slice location along the $z$-direction. The bacteria detection is performed by the level set method described in sec. 3.5. We take advantage of the parallel layer configuration to identify the slices belonging to each layer. As it can be seen in Fig. 3.2, while the number of detected cells is fairly constant within a layer, in the transition slices, the number of detected cells falls due to the mutual layer encroaching. We use these dips to identify the first and last slice of each layer.
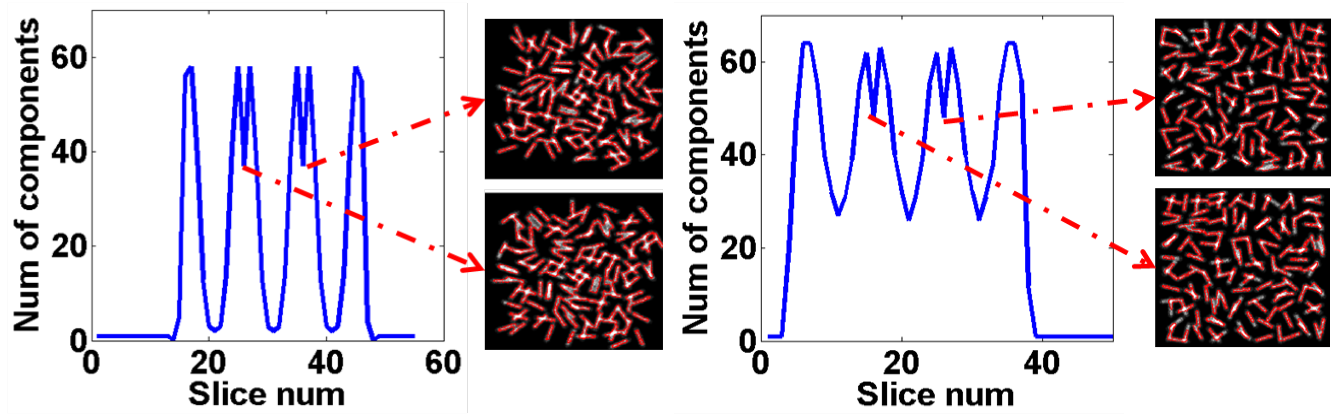

FigURE 3.2. Automated layer detection examples.

Once the starting slice is selected, the individual bacteria can be identified and the skeleton of the background used as a prior in the segmentation of the following slice (Fig. 3.1 a). The algorithm, highlighted in Fig. 3.1 (b-h) and described in the following sections, will identify ideal seed locations for each 
bacteria (Fig. $3.1 \mathrm{~b}$ ), evolve level set functions from the seed point (Fig. $3.1 \mathrm{c}$ ) and determine a stopping criterion (Fig. $3.1 \mathrm{~b}$ ) based on the skeleton of the background [42] (Fig. 3.6 b).

\subsection{Curvature-based seed selection}

After smoothing the image, a set of local maxima to use as initialization seed for the level set analysis is found by evaluating the Hessian [43] of the original image and using the most negative eigenvalues to select the brightest locations with the highest curvature within each bacterium [40].These local maxima define the seeds of the initialization contours (Fig. 3.3 and Fig. 3.4 a) for the level set evolution (Fig. $3.1 \mathrm{~b}$ ).
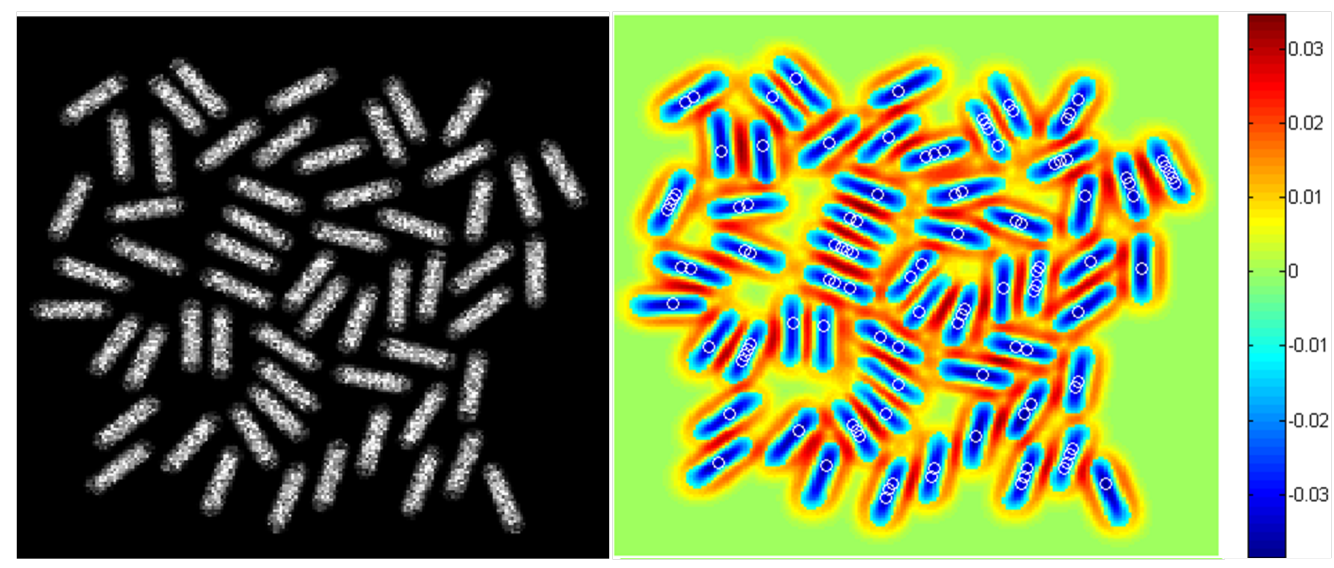

FIGURE 3.3. Example of seed selection.

\subsection{Iterative level set evolution}

The local level set method developed here is motivated by the idea of oversegmentation. In [28], the authors proposed a method for over segmentation by evolving a level set function from local uniformly spaced seeds points. In our method, we evolve the level set function from the seeds identified as discussed 
in sec. 3.3 and allow the contours, defined by the level set function, to evolve in a local neighborhood. The level set is evolved until it reaches a stopping criterion, obtained exploiting the prior slice segmentation result. This process is shown in Fig. $3.4 \mathrm{a}-\mathrm{c}$ ).

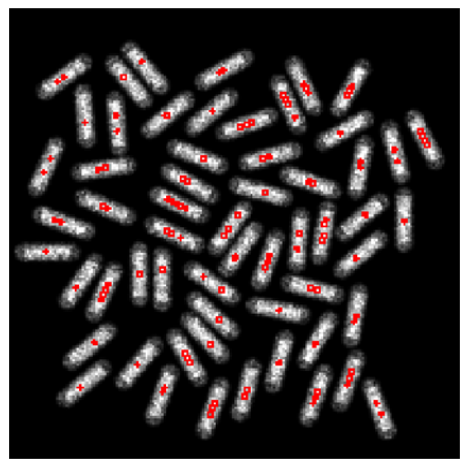

(a)

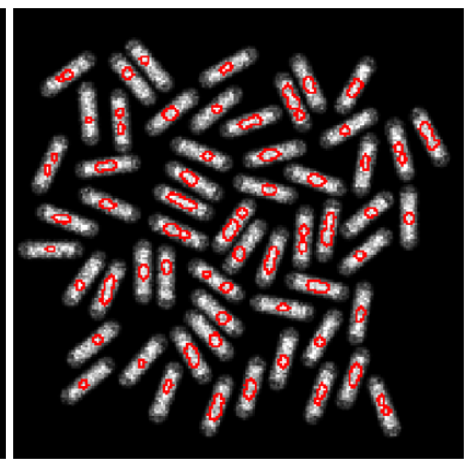

(b)

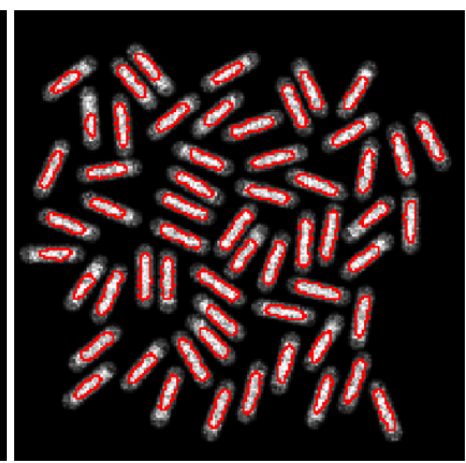

(c)

FiguRE 3.4. Seed selection and level set evolution. (a) Place initial curvature based seeds. (b) Evolving level set (curve) specified in section 3.5. (c) Multiple seeds in one cell will merge in restricted region during the evolution.

The framework of level set segmentation was proposed in [36]. As mentioned in the previous chapter, the object boundary is defined by the zero level set, where $\phi$ is a signed Euclidean distance function

$$
C=\{(x, y): \phi(x, y)=0\}
$$

In this method, the inside of the contour is denoted by $\phi<0$, while the outside is $\phi>0$. The gradient descent update for the discrete level set at each time step $\Delta t$ is given by

$$
\phi^{n+1}=\phi^{n}-\mathrm{V}\left|\nabla \phi^{n}\right| \Delta t
$$

To prevent the merging of evolving contours in slices where the boundaries of adjacent bacteria blur into each other due to the imaging resolution, it is critical to define a stopping criterion (where the curve evolution velocity is 
set to 0 ) that clearly separates adjacent cells. This is achieved by defining a piecewise velocity function as follows:

$$
V=\left\{\begin{array}{cl}
0 & , \text { if } \mathrm{SC}=1 \\
g[1-\epsilon \kappa]-\beta \nabla g \mathbf{N}, & \text { otherwise }
\end{array}\right.
$$

The $V=0$ condition effectively prevents the contours, driven by the level set evolution, from crossing the boundaries defined by the stopping criterion (SC) described in sec. 3.7. The velocity model $V=g[1-\epsilon \kappa]-\beta \nabla g \mathbf{N}[44]$ represents the propagating rate of the evolving curve toward the cell boundary. In this formula, $\kappa$ is generally the curvature forcing a slow down at the boundary areas, $\epsilon$ and $\beta$ are the contour smoothing and edge attracting regularization, and $g$ is the local affinity function as defined in [28]:

$$
g(x, y)=e^{-E(x, y) / v}, E(x, y)=\frac{|\nabla I|}{G_{\sigma} *|\nabla I|+\gamma}
$$

where $\nabla I$ is the gradient of the image, $G_{\sigma}$ is a smoothing kernel, and $\gamma$ is a constant to guarantee that $E$ will remain limited in the presence of small gradients.

\subsection{Localization of individual bacteria}

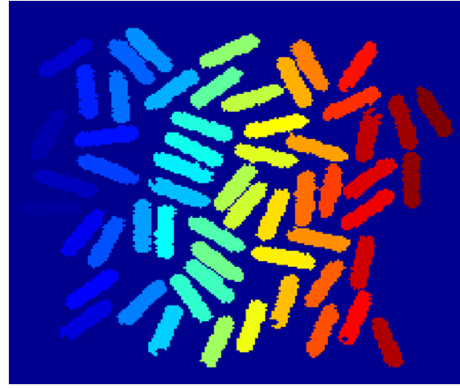

(a) Contour clean up

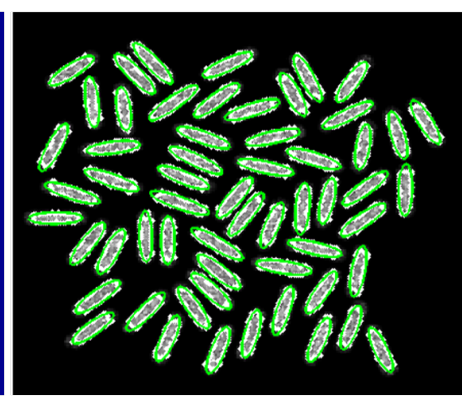

(b) Ellipse fitting

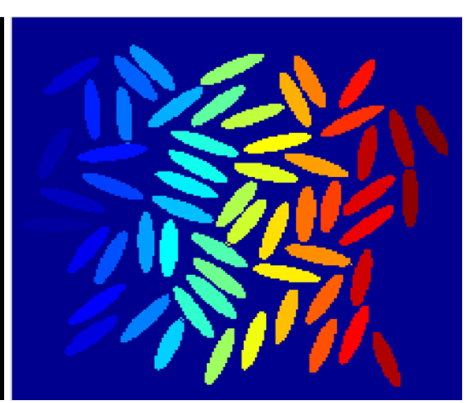

(c) Identification

FIGURE 3.5. Example of identification of individual bacteria.

The final signed distance function $\phi$ is first binarized, followed by component labeling to identify and count the number of bacteria. To extract information about the individual bacteria, the final contours of each bacterium 
are fitted with an ellipse $\left(a x^{2}+b x y+c y^{2}+d x+e y+f=0\right)$ using the leastsquare method described in [38] with an added constraint based on our prior knowledge of the average bacterium size. Location, length, and orientation are then evaluated from the estimated conic equation parameters $a, b, c, d, e, f$.

\subsection{Stopping criterion and re-initialization}

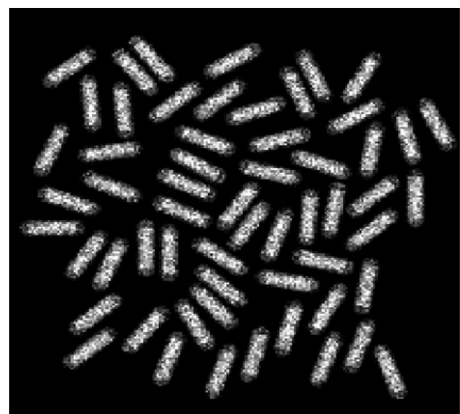

(a)

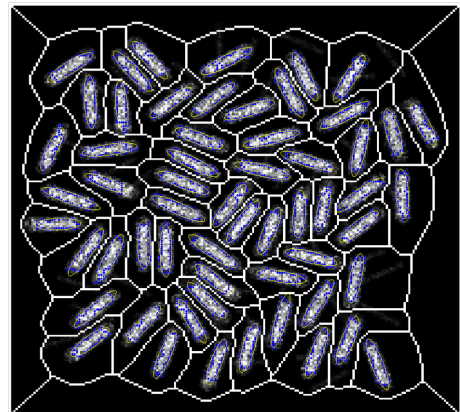

(d)

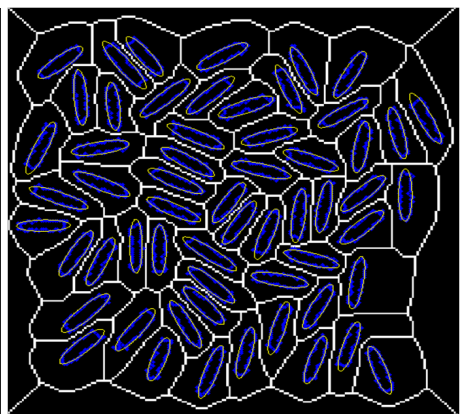

(b)

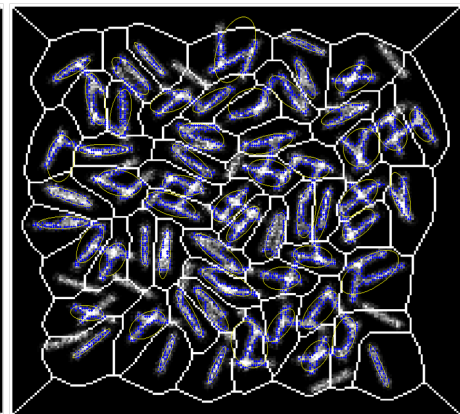

(e)

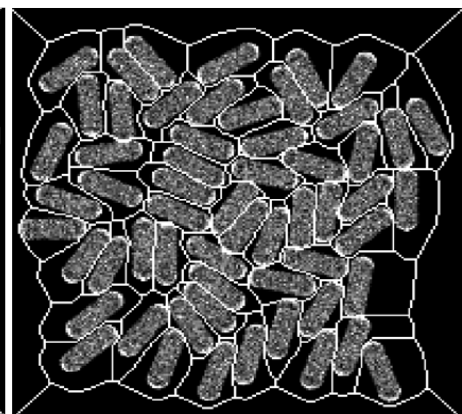

(c)

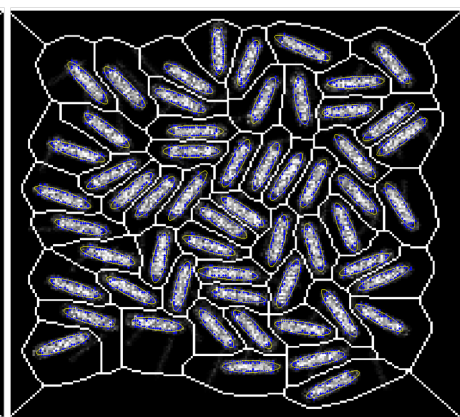

(f)

FIGURE 3.6. Set background stopping criterion by calculating the skeleton of the background. (a) is original image. (b) shows the stopping criterion (white) set as the skeleton of background that precludes ellipses (yellow), which is the least square fitting of the level set contours (blue). (c) shows that the stopping criterion is efficient to separate most situations in a image such as parallel touching cells. The reinitialization of the skeleton is shown from (d) one slice before the layer change (e) to (f) one slice after the layer change. 
Following the ellipse fitting, the elliptic contours are used to define the bacteria-background boundaries. Taking advantage of the smoothness of these boundaries, we can in turn obtain a smooth skeleton of the background region (Fig. $3.6 \mathrm{~b}$ ). This skeleton provides the additional stopping criterion that forces $V=0$ in our piecewise definition of $V$ (Eq. 3.3), and is used as a prior information to insure that the separation between the bacteria is maintained, even in the presence of blurred edges, during the level set evolution step in the following slice.

At the interface between layers (sec. 3.3), the skeleton from the previous slice is disregarded and a new skeleton is automatically reinitialized to conform to the location and orientation of the detected bacteria in the new layer (Fig. 3.1f). The process is shown in Fig. 3.6 (d-e). 


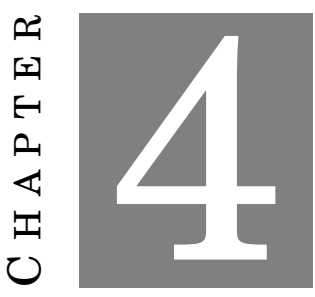

\section{EXPERIMENTAL RESULTS AND ANALYSIS}

$\mathrm{T}$

his chapter presents the results of the segmentation proposed in this thesis. The approach is analyzed in terms of parameter sensitivity, computational expense, program complexity evaluation and comparing segmentation performance with other methods. In addition, some other analysis, such as the effect of different super-resolution factors, are also discussed in the later sections of this chapter.

\subsection{Dataset and parameter set up}

To test the performance of proposed BACT-3D method on multi-layered biofilms, five simulated data sets (see table 4.1) were generated as described in sec. 3.2. To evaluate the performance of the algorithm as function of the instrumentation imaging resolution, two achievable super-resolutions were simulated in each dataset: the first is approximately a two-fold improvement in resolution over diffraction-limited imaging (called Resolution 1) and the second is three times better (and is called Resolution 2). The first dataset was generated by utilizing a Gaussian with a kernel half the size of the diffraction 
limited as specified in section 3.2 whereas the second used a kernel one third of the diffraction limited size.

TABLE 4.1. Multi-layered biofilms datasets.

\begin{tabular}{ccc}
\hline Image name & Image size & Num of cell \\
\hline data 1 & $201 \times 201 \times 55$ & 174 \\
data 2 & $201 \times 201 \times 49$ & 147 \\
data 3 & $201 \times 201 \times 51$ & 192 \\
data 4 & $201 \times 201 \times 51$ & 192 \\
data 5 & $201 \times 201 \times 51$ & 192 \\
\hline
\end{tabular}

Each slice is analyzed separately and the regions corresponding to each bacterium are joined across slices to form the final 3D reconstructed volume. To separate the individual layers in the $z$-direction, the slices that are detected as layer transitions are set to zero value. Besides, the selection of parameters in the algorithm is shown in table 4.2. The radius of seed is set to be one pixel. $\alpha, \epsilon$ are chosen for better leakage and smoothing balance, which ensures the contour not across the cell boundaries and not exclude the valid areas in the bacteria. Time step $\Delta t$ is set to be 1 which is neither too small to slow down the speed of the curve evolution nor too large to skip the proper edges. These parameters are chosen carefully evaluating the segmentation performance with different parameter values, where the best quantity and the most accurate segmenting areas are achieved.

TABLE 4.2. Parameter settings for BACT-3D on multi-layered datasets.

\begin{tabular}{cc}
\hline Parameter & Value \\
\hline Radius of Seed & 1 pixel \\
$\alpha$ & 0.3 \\
$\epsilon$ & 1 \\
$\Delta t$ & 1 \\
Max number of iterations & 1000 \\
\hline
\end{tabular}




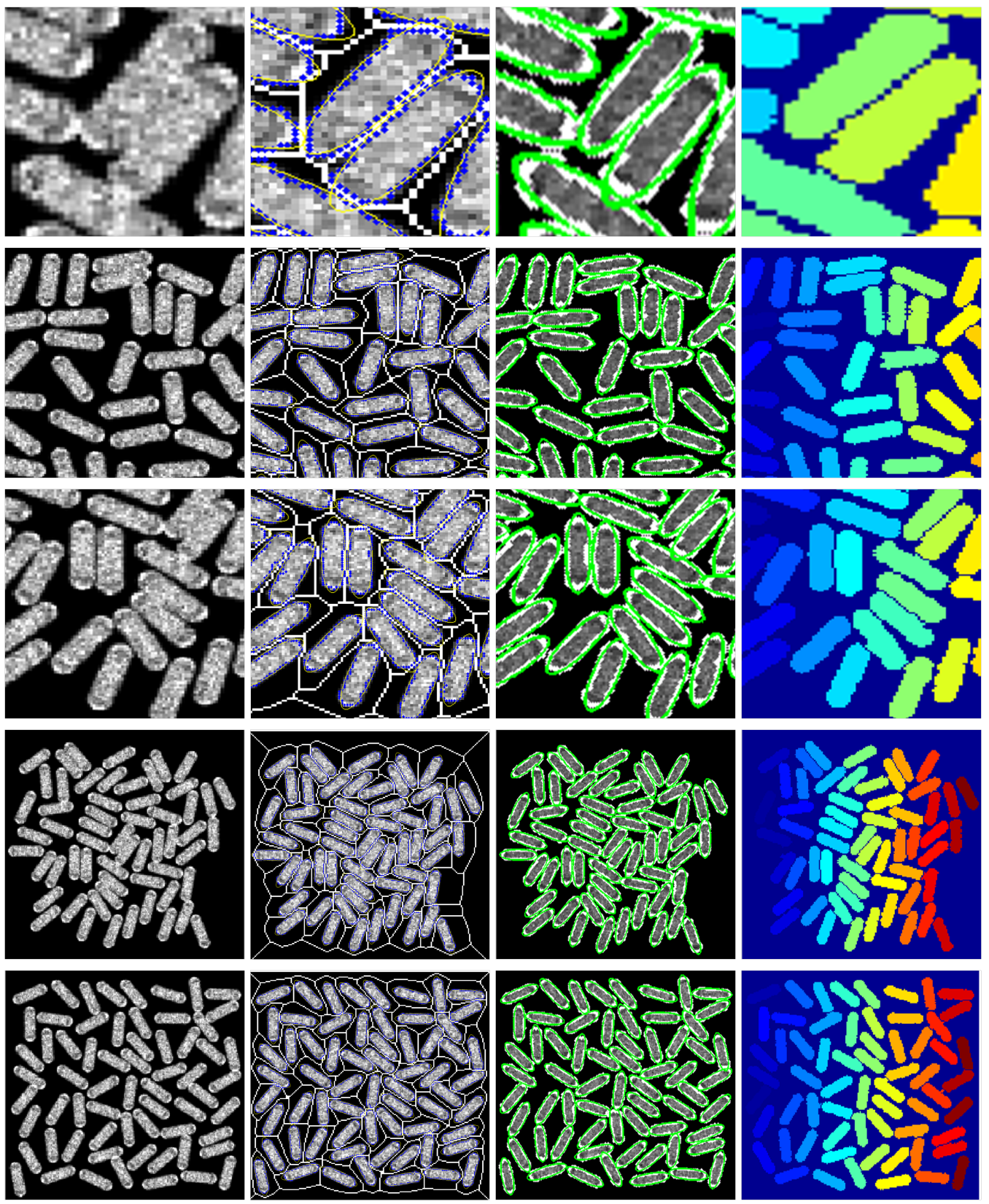

FIGURE 4.1. Sliced results of multi-layered biofilms. Column 1: original images. Column 2: background stopping criterion (skeleton) in white. Column 3: the results after ellipse fitting in green. Column 4: labeling of bacteria. 


\subsection{Experimental results}

The experimental results exhibit efficient segmentation performance on multi-layered bacterial biofilms using Bact-3D. Some of the sliced results are shown in Fig. 4.1. It is observable that all the bacteria are isolated with the cell walls defined by skeleton of ellipse-smoothed background (the background excludes the bacteria areas fitted with ellipses, see section 3.7). Bact-3D obtains the 3D reconstruction of multi-layered bacterial biofilm by stacking up sliced segmentation results (Fig. 4.2) along the growing of $z$-axis (results are shown in Fig. 4.3).

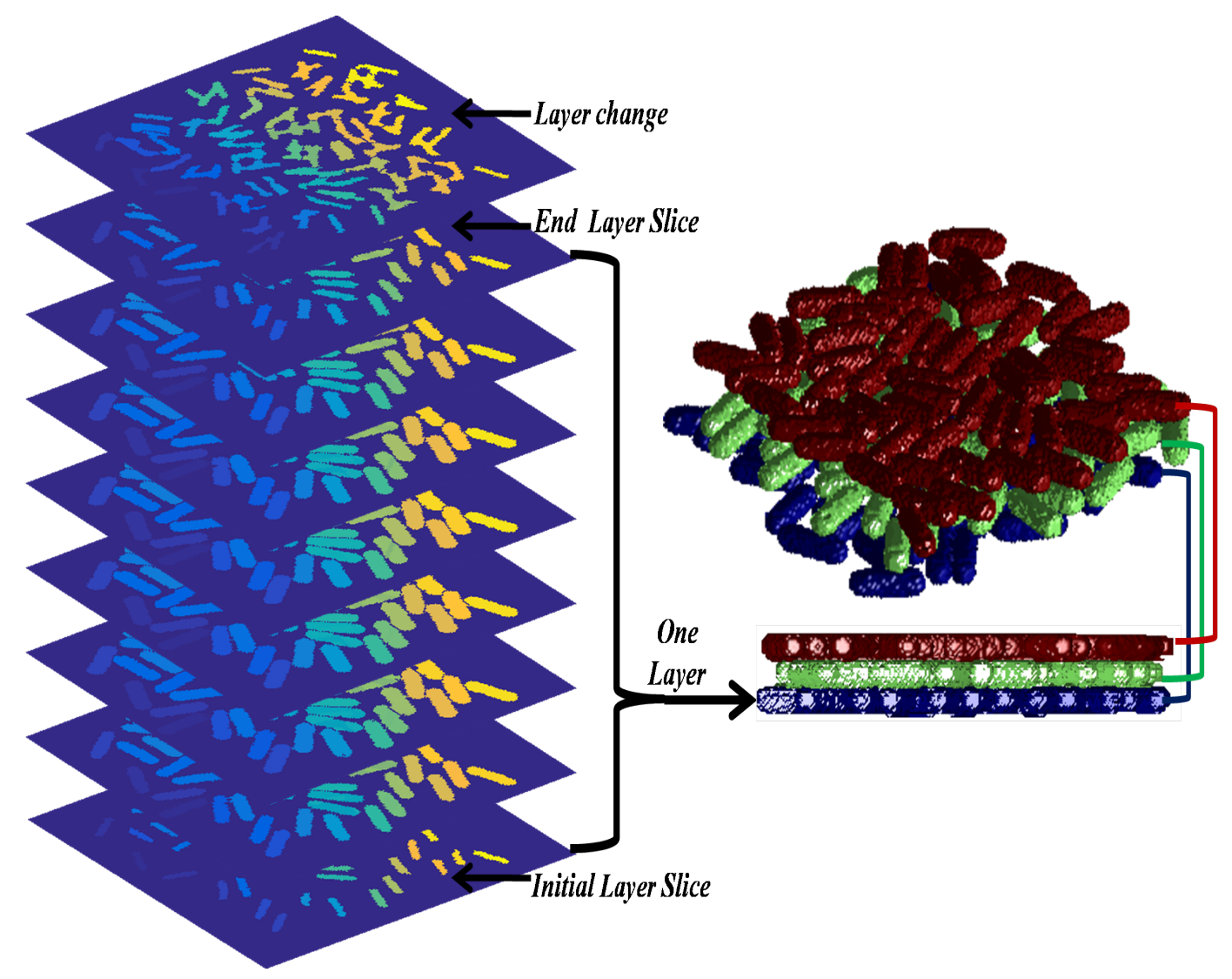

FigURE 4.2. Reconstruction of 3D biofilm. 

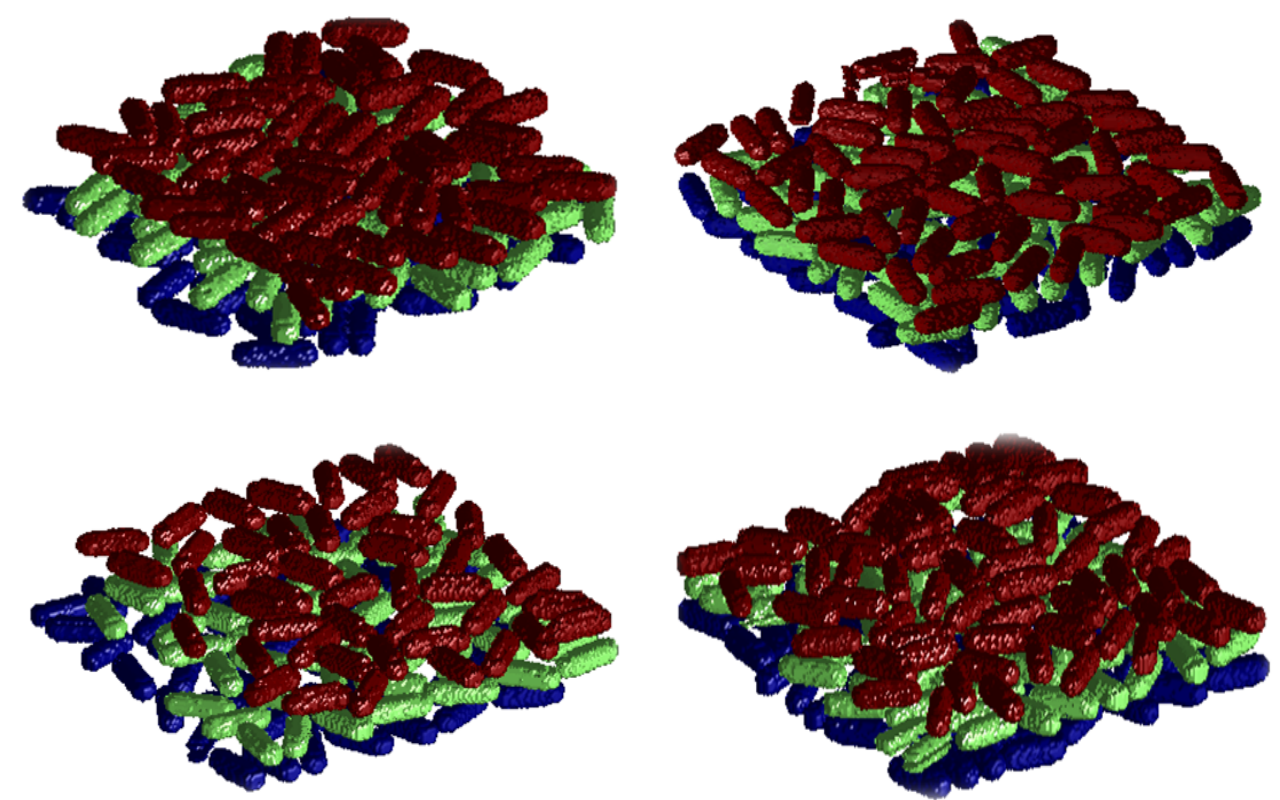

FIGURE 4.3. Results of reconstructed 3D multi-layered biofilms.

There are two features showing in above experimental results of Bact-3D segmentation approach:

(a) Locality: the contours are always limited to single-cell regions which ensures the separation between each layer and each bacterium;

(b) Trackability: locations and orientations are available for each individual bacterium, which will allow to track the cellular behavior of bacteria in future work.

\subsection{Comparison of segmentation performance}

We quantitatively compared our results with three published algorithms: single cell tracking by Yan, et al. [41], Chan-Vese [25] and L2S [30]. Yan's single cell tracking method is implemented using the available open source code where the value of 10 , used in the watershed algorithm, was empirically 
chosen to obtain the optimized the output with best segmentation performance. Chan-Vese was implemented using the 3D implementation by Zhang [45] with a parameter selection of image weight $=100$ and smooth weight $=0.1$. For L2S, we used the published code in a slice-by-slice implementation and then merged all the segmented slices along the $z$-direction to achieve the final 3D volume.

Dice coefficient (Dice), mean squared error (MSE) and cell detection accuracy (CD) are used to quantitatively evaluate the performance of these three methods (Table. 4.3 and Table. 4.4). The Dice coefficient compares the similarity between two sets: the ground truth $\mathrm{V}_{\mathrm{g}}$, which is the binarized volume with the areas inside of the ground truth bacterial biofilms setting to be 1's, and the detected $V_{t}$ volume. It is computed using

$$
\text { Dice }=\frac{2\left|V_{g} \cap V_{t}\right|}{\left|V_{g}\right|+\left|V_{t}\right|}
$$

where $|\cdot|$ denotes the cardinality of the corresponding set. Dice varies between 0 to 1 with unity indicating optimal segmentation.

Mean squared error measures the average squared errors between ground truth $V_{g}$ and detected $V_{t}$ volumes:

$$
\mathrm{MSE}=\left\|V_{g}-V_{t}\right\|_{2}^{2}
$$

The MSE is normalized by the total number of pixels in the ground truth volume.

The cell detection accuracy (CD) determines how many cells are segmented and identified $\left(N_{t}\right)$ compared with the actual number in the ground truth volume $\left(N_{g}\right)$. We define this measure similarly to the Dice coefficient:

$$
\mathrm{CD}=\frac{2 \min \left(N_{g}, N_{t}\right)}{N_{g}+N_{t}}
$$

The ground truth was defined, identically for all the resolutions, as the solid volumes occupying the simulated bacterial cells before the blurring by the Gaussian kernel. The value was set to unity for the inside of the cells and zero for the outside. The same index-type function was used for the detections obtained by the three methods allowing for a direct comparison. 

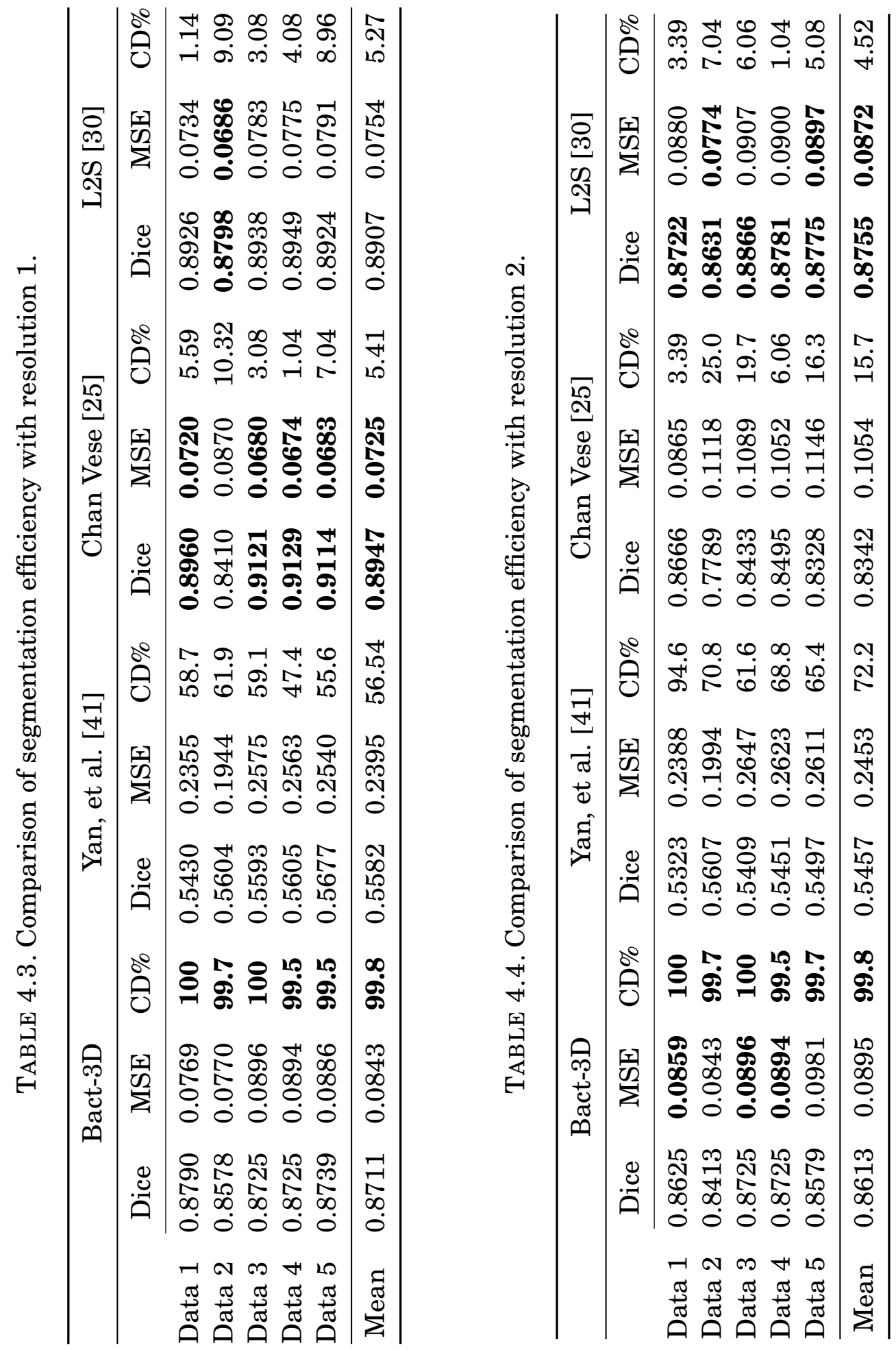

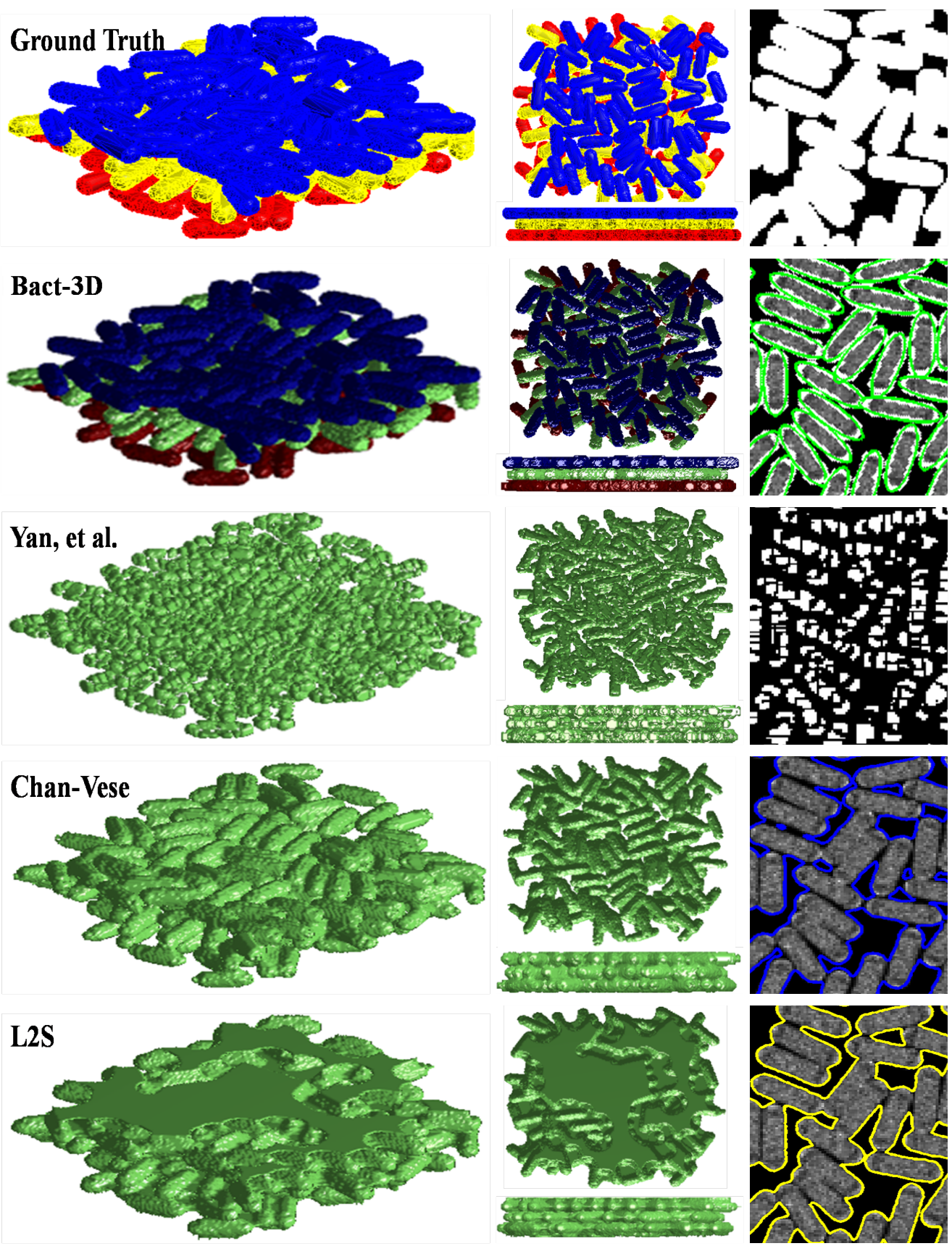

FigURE 4.4. Comparison of segmentation algorithms. The first and second column show final 3D volumes reconstructed by each algorithm from different viewpoint (different color for each layer). The third column shows the segmentation on an individual slice. 
Due to the very high over-segmentation results, the single cell tracking method [41] has no evident advantages when compared with the other three methods. Although L2S [30] and Chan Vese [25] show a slightly better performance in the Dice and MSE metrics, when considering the accuracy in single cell identification (CD), the proposed Bact-3D shows a significant advantage with an average accuracy of $99.81 \%$. Bact-3D output provides us not only with a clear 3D reconstruction of the layered biofilm (Fig. 4.4, column 1 ) but also with the ability to identify each individual bacterium allowing the extraction of statistical information about location, size and orientation. Fig. 4.4 shows an overall visualized comparison of these four methods.

\subsection{Algorithm complexity analysis}

The complexity of Bact-3D is analyzed by evaluating the worst case function behavior corresponding to size of input argument, denoted by $\mathscr{O}$. Suppose the input 3D image size is $n \times n \times m$ and the cell number can be detected in each layer is $k$. Then the analysis can be divided into three parts.

The background initialization part is approximately linear to the input total pixel numbers $(N=n \times n)$ in each $z$-slice. Layer detection is just the evolution of level set, where the complexity is $\mathscr{O}(N)$ [28]. Then background smoothing step, which is the localization of individual bacterium, is realized by calculating the least square fitting of each detected cell (in total is $k$ cells). Suppose numbers of the pixel on the boundaries of each cell are $N_{1}, N_{2}, N_{3}, \ldots, N_{k}$. Then, the complexity of this step is $\mathscr{O}(k) \bullet\left(\mathscr{O}\left(N_{1}\right)+\mathscr{O}\left(N_{2}\right)+\mathscr{O}\left(N_{3}\right)+\ldots+\mathscr{O}\left(N_{k}\right)\right)$. Because $N_{1}, N_{2}, N_{3}, \ldots, N_{k}$ are much smaller than $N$, the complexity is approximately $\mathscr{O}(k N)$. The computation of background stopping criterion (skeleton) is $\mathscr{O}(N)+$ $\mathscr{O}(p \log p)$ [42], where $p$ is the pixels inside of the thinning volume. In practice, $k$ and $p$ are also much smaller than $N$, so the complexity by now is roughly approaching $\mathscr{O}(N)$.

The main part of iterative layer segmentation also starts from level set evolution $(\mathscr{O}(N))$ with defined stopping criterion. Cell clean and label processes 
are linear with pixel size of the input $(N)$. The complexity of the ellipse fitting part and definition of the background stopping criterion are both $\mathscr{O}(N)$. The re-initialization of the skeleton depends on layer detection, which is also $\mathscr{O}(N)$ computations.

Finally, considering the total iterations of the algorithm, which is the number of $z$-slice (m), the overall computation complexity is $\mathscr{O}(m N)$. In other words, the complexity is roughly linear with the total number of voxels ( $n \times$ $n \times m)$ in the input volume.

\subsection{Operating time evaluation}

TABLE 4.5. Operating time of Bact-3D.

\begin{tabular}{cccc}
\hline Function & Calls & Total Time (sec) & RTO (\%) \\
\hline bact3d & $\frac{1}{1}$ & $\underline{627.154}$ & $\underline{100.000}$ \\
layer detection & 31 & 184.956 & 29.491 \\
ellipse fitting & 35 & 168.903 & 26.932 \\
level set evolution & 4 & 44.092 & 7.030 \\
background initialization & 35 & 0.822 & 0.131 \\
seed selection & 31 & 0.581 & 0.093 \\
cell label and clean & & &
\end{tabular}

Table 4.5 reflects the operating time of Bact-3D with an input of $201 \times 201 \times$ 55 voxels. The first column identifies the core functions. The second column is the number of calls times, which is determined by the layer numbers detected along $z$-direction in the volume. Third column is the total function time. The fourth column indicates function running time occupation (RTO), calculated by:

$$
\mathrm{RTO}=\frac{\text { function operating time }}{\text { total time }}
$$

One drawback of this proposed method is the execution time, which is expected to increase when dealing with larger input 3D volumes. There are four dominant functions: layer detection, ellipse fitting, level set evolution 
and background initialization. Layer detection is the function to divide the whole stack into several layers, which is a pre-step for local geometric contour. The problem here is that layer detection does the whole process of level set evolution before the main part of the algorithm, which cost most of the time. An alternative solution to improve this automated layer detection could be embedding this process into each $z$-slice iteration. For instance, we can evaluate the similarity of bacteria positions in neighboring slices and re-initialize the stopping criterion if there are detected changes. In this case, the operating time would be reduced by $1 / 3$. Another costly computation is ellipse fitting, which is used to smooth the background. One way to decrease the time could be fixing the ellipses where the positions of cells are not changing, and re-fitting the ones that change the positions and orientations.

\subsection{Discussion}

Overall, Bact-3D presents an efficient solution to segment dense multilayered bacterial biofilms based on geometric active contour. The segmentation exploits super-resolution data achieved via novel microscopy and provides a robust precondition for tracking [46] the bacteria growth in future work.

However, the accuracy of segmentation decreases when bacteria become randomly distributed in 3D space. Segmentation performance for these random distributed data using Bact-3D is not as good as for parallel layers (Fig. 4.6), because layer transition is no longer predictable ( a failure of layer detection is shown in Fig. 4.5). When we used the information of upper slice in $z$-direction to set up the stopping criterion for active contour, new emerging cells will be mis-cut or ignored, as show in Fig. 4.6, because cells are no longer in the same horizontal plane. Moreover, it gets worse when working with real dataset (Fig. 4.6). There are two main problems during the segmentation period using Bact3D. One is that there is not clear one slice where all the cells are perfectly separated from each other. The other problem is that the variant intensities inside of the noisy image exhibit low contrast.

Under these circumstance, we need to improve and extend our local level 
set algorithm to random-distributed data sets with noise to increase the robustness of our method.

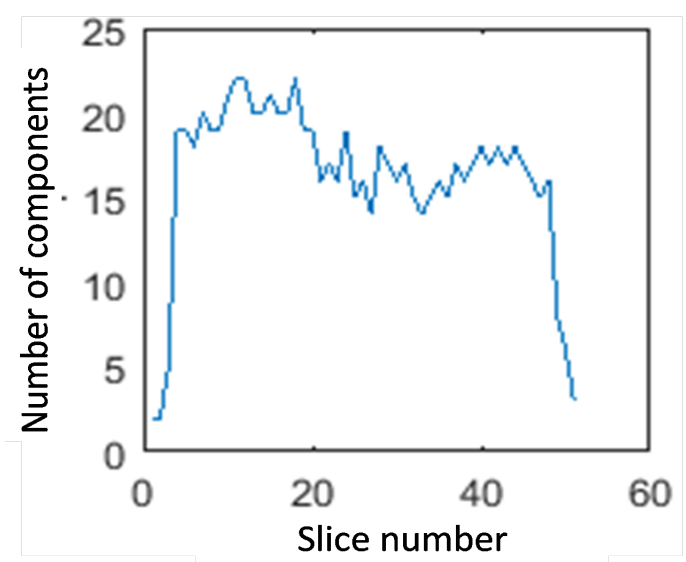

FIGURE 4.5. A failure layer detection situation of Bact-3D.
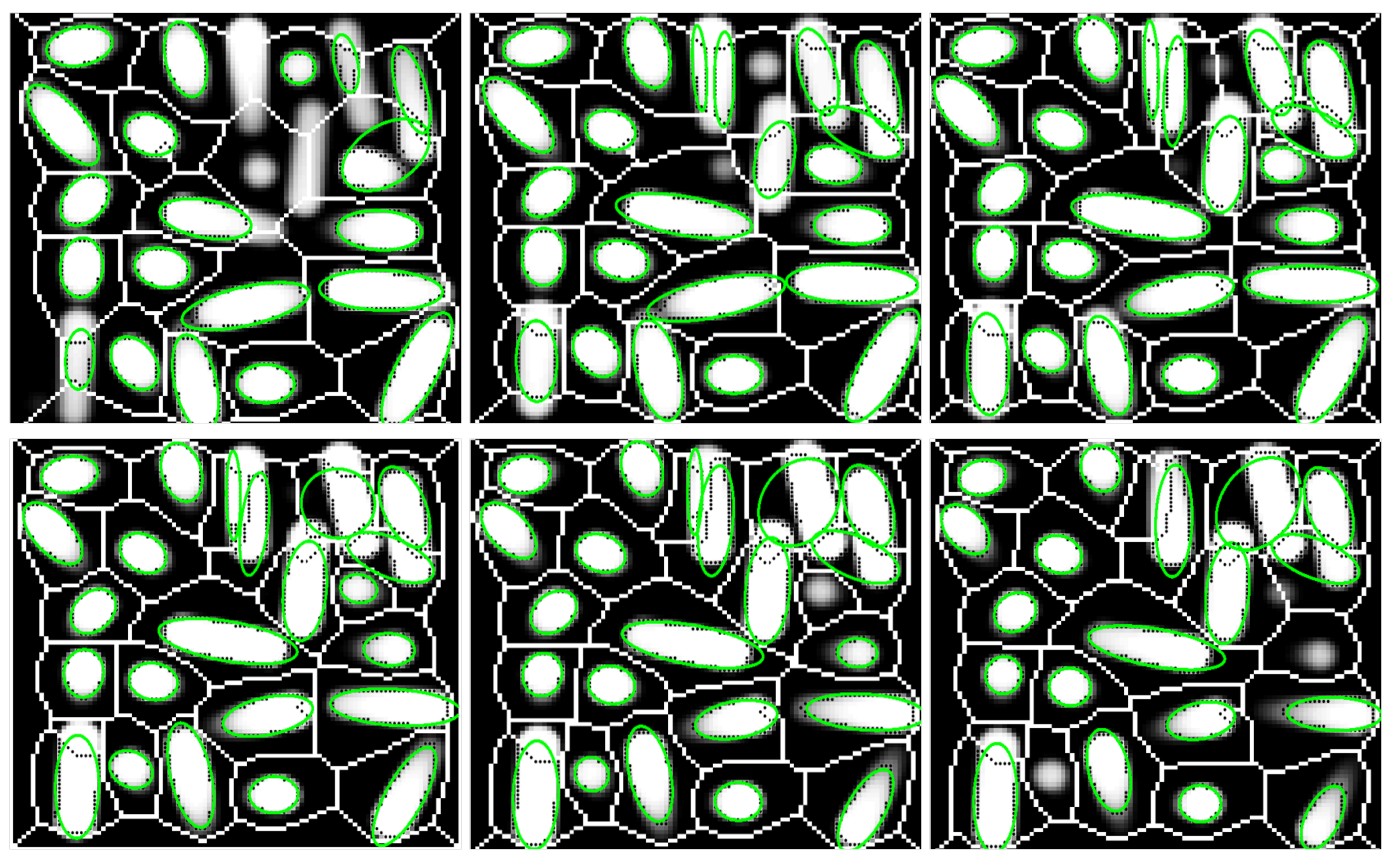

FIGURE 4.6. Example of the limitations of Bact3D (simulated data). Continuous sliced performance on random distributed uniform intensity $3 \mathrm{D}$ image is shown. (slice 41 to slice 46 from left to right, up to down) 

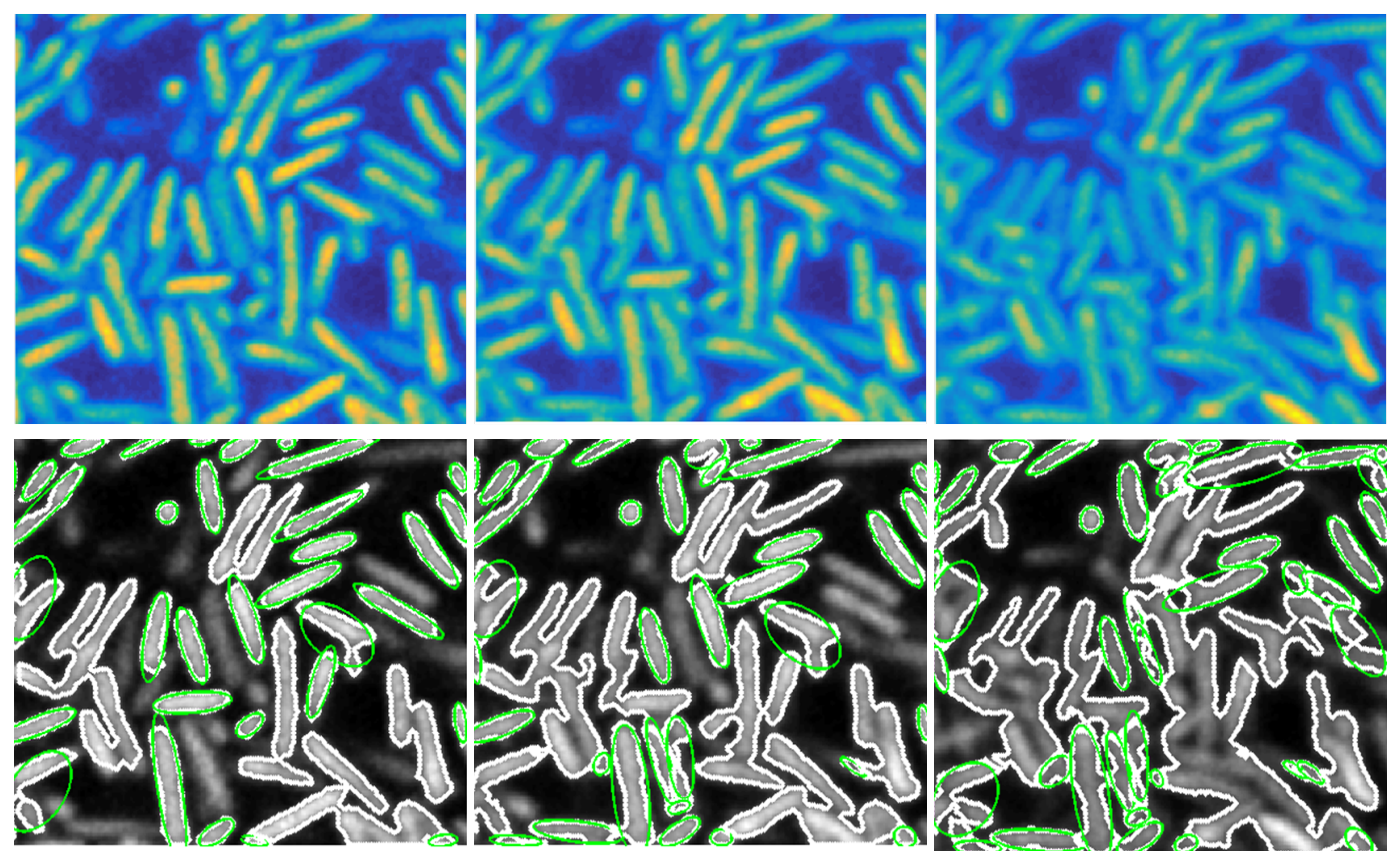

FIGURE 4.7. Example of the limitations of Bact3D (real data). Continuous sliced performance on real 3D image subset is shown (slice 1 to slice 3 from left to right). First row are original real images. Second row are segmentation results using Bact3D. The cell detection rate is decreased to about $50 \%$ 



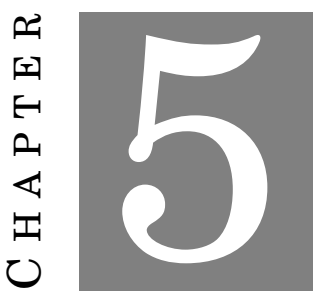

\section{IMPROVEMENT AND FUTURE WORK}

\footnotetext{
7 onsidering the limitations of Bact-3D when we extend the dataset to a more general situations of random distributed datasets, an improvement of the algorithm is necessary. There are two main challenges in which the problem: one is the difficulty in finding the well-segmented slice (maximum number of the cells are separated) along $z$-direction in the 3D volume, where the cells are overlapping randomly; the other one comes from the influence of noise and the resolution limit, which decrease the quality of raw images as well as the accuracy of segmentation. To rise to these challenges, several efforts are briefly introduced and discussed in this chapter. Meanwhile, more improvements are underway for future work.
}

\subsection{From active contour to active surface}

Because of the lack of clear separation along the $z$-direction between layers, the ellipsoidal structure in one single slice is not sufficient to separate individual bacterium in a randomly distributed dataset. However, the overall structure of bacteria in space still motivates the idea that, if we step through 
several layers of the bacteria, the preserved ellipsoidal structure could still lead to the correct boundaries. An intuitive example of this improvement is shown in Fig. 5.1. The upper two cells simulated the layout of original dataset where the cells are distributed within the same vertical layer. The lower three cells represent overlapping cells in the new simulated datasets, where the cells are randomly distributed in the $3 \mathrm{D}$ space. The red lines are the boundaries we are trying to extract by our improved method. The orange markers are chosen to be the start of the evolving curve/surface.

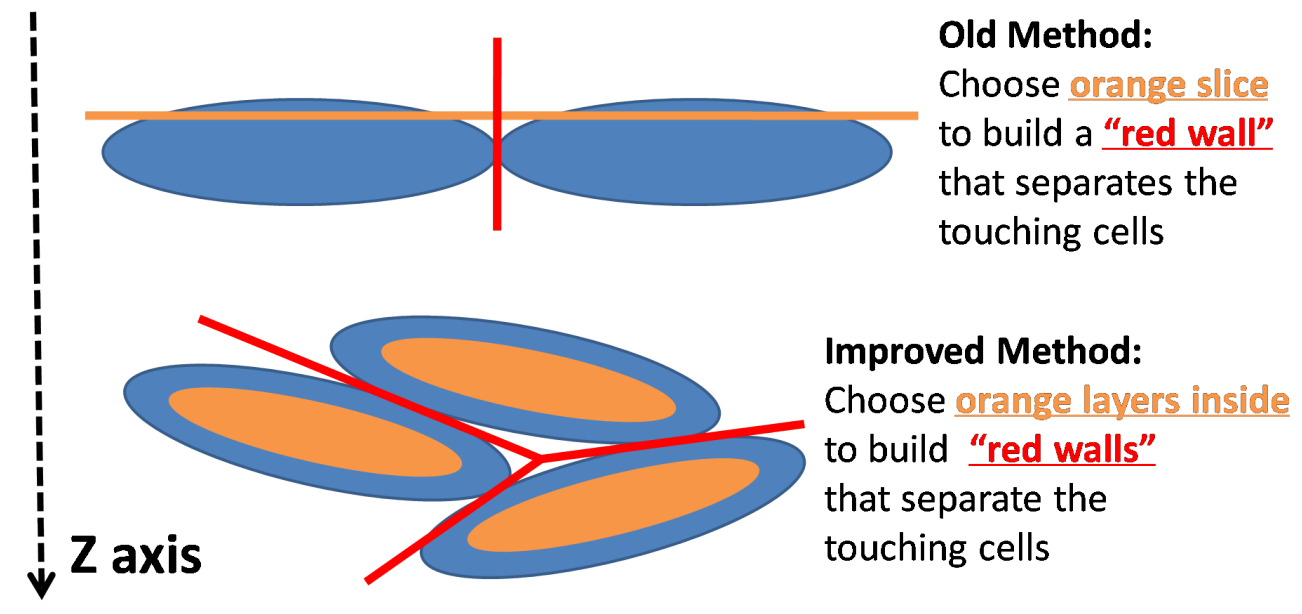

FIGURE 5.1. Demonstration of core innovation designs in improved algorithm: Bact-3Ds.

To identify the cells' boundaries for the non-layered configuration, we propose Bact-3Ds to directly build a geometric active surface model (deformable model) in 3D instead of evolving the level set (active contour) in each slices and stacking them up. Following the core idea in Bact-3D, the active surface is initialized with selected seed (section 5.1.1). Then, the surface, defined by the zero level set of $4 \mathrm{D}$ signed distance function (section 5.1.2), evolves until it reaches either boundary of the cell or boundary of the single-cell region (section 5.1.2). A preliminary result of this new model is shown in section 5.1.3. 


\subsubsection{Distance-based seed selection}

The seed is the orange part in the lower case in Fig. 5.1. It is chosen by applying the 3D Chan-Vese algorithm [25] to the active surface directly on the raw image volume. As introduced in chapter 2 (section 2.2.2), the Chan-Vese algorithm classifies the image into two regions, background and foreground (bacteria), evaluating the difference in intensities. This algorithm defines a $4 \mathrm{D}$ signed distance function $\phi(x, y, z ; t)$, which gives an initial segmentation with zero level set marking the boundary of bacteria in 3D. At this point, cells would be still connected at the zero level set, but the inner parts (marked with higher distance values in our case) of each cell are still separated (Fig. 5.2 a). The distance threshold is chosen such that it preserves the maximum number of cells counted in the 3D volume and the regions are selected as seed (Fig. 5.2 b).

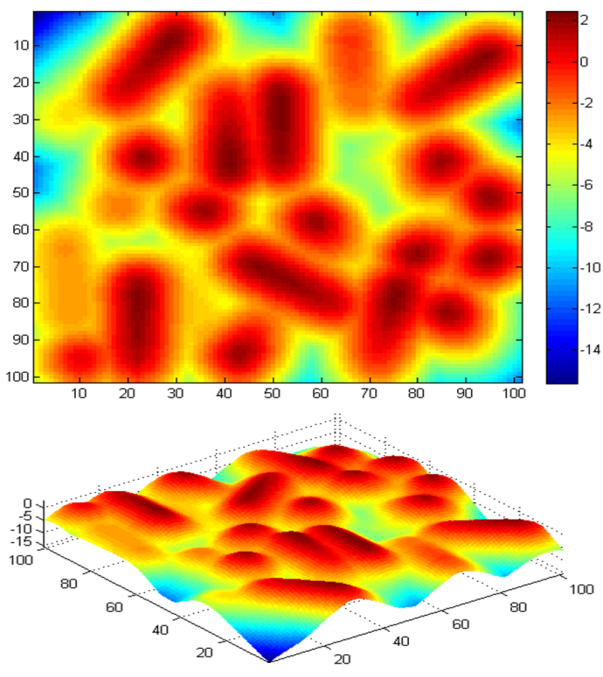

(a)

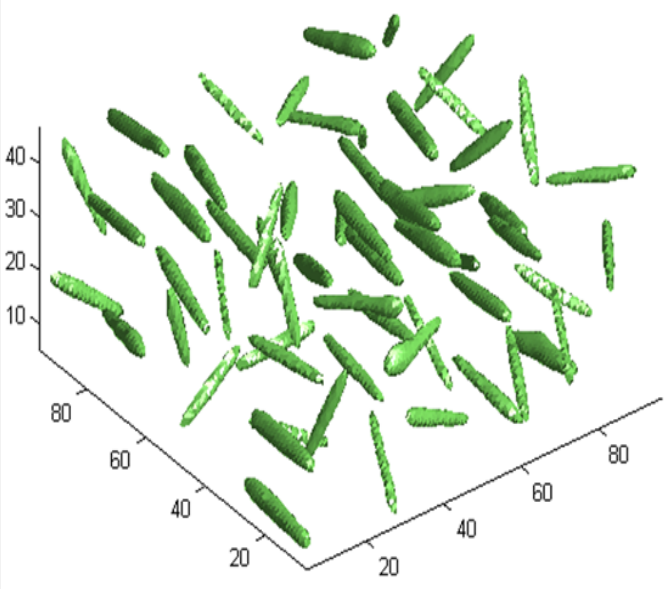

(b)

FIGURE 5.2. Distance-based seed selection. (a): 4D signed distance function after the evolution of the Chan-Vese Algorithm [25] in one slice (upper image: top view; lower image: $3 \mathrm{D}$ viewpoint). (b): The seed is obtained by choosing the dark red parts (higher distance values) in (a). 


\subsubsection{Curvature-based active surface model}

After the initialization of the 3D level set to identify the seed, the zero level set is evolved until it reaches specific positions which are the boundaries of single-cell regions.

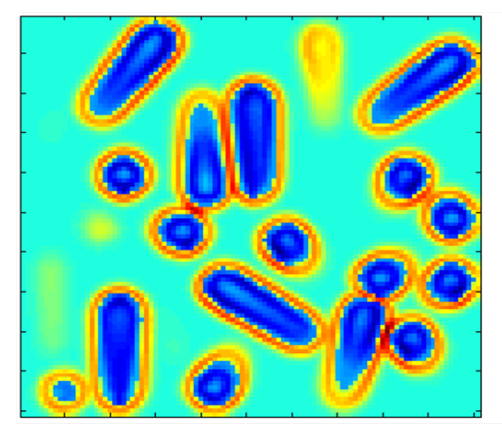

(a)

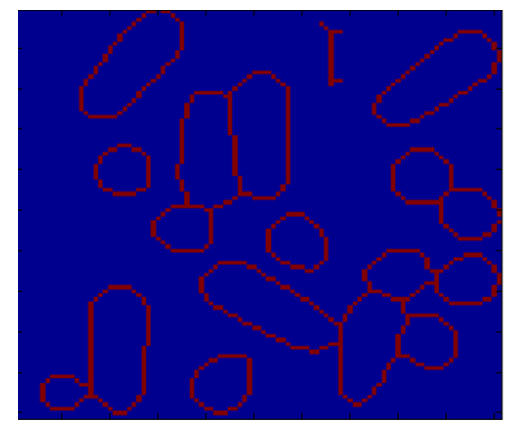

(c)

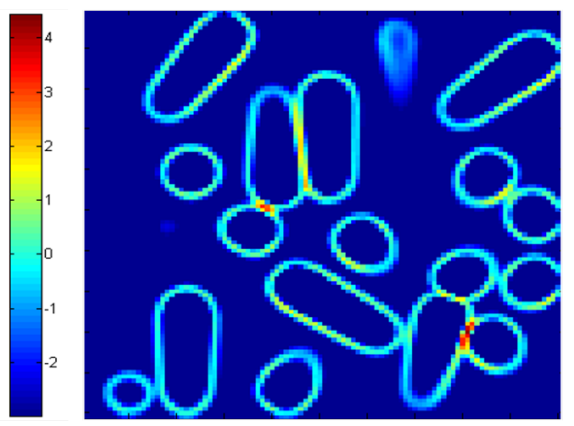

(b)

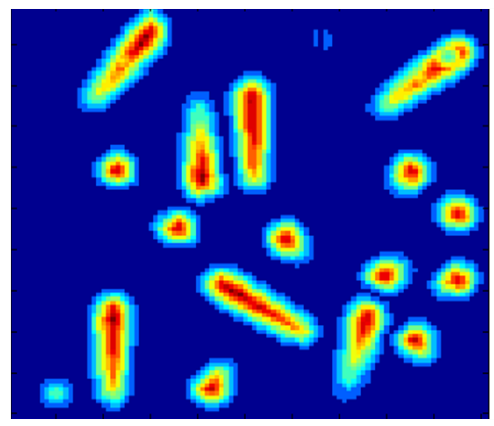

(d)

FIGURE 5.3. Velocity design of curvature-based active surface model.

(a) One slice of the 3D curvature-enhanced image. (b) Gap regions with highest curvature values. (c) Skeletonizing the volumes between each cells in $3 \mathrm{D}$ could give the skeleton of these gaps in $2 \mathrm{D}$ images. (d) Distance velocity field (DVF).

Same as Bact-3D, these single-cell regions are defined by skeletonizing the gaps between each cell to the minimum width ( 1 voxel in this case). The gaps are enhanced evaluating maximum eigenvalue of the Hessian of $3 \mathrm{D}$ volume, where the intensity transitions (gaps) have the maximum curvature features (Fig. 5.3 a). Then, choosing these enhanced gaps (Fig. $5.3 \mathrm{~b}$ ) and thinning them to the minimum width (Fig. $5.3 \mathrm{c}$ ) from three directions $(x, y, z)$ could 
build up a closed skeletonized surface around each bacterium ensuring the evolution of seeds never exceed these closed single-cell regions.

In the actual implementation (review equation 2.5 in sec. 2.2.1), this singlecell region is demonstrated by designing the velocity of $3 \mathrm{D}$ level set evolution as an outward distance velocity field (DVF) (Fig. $5.3 \mathrm{~d}$ ). We define the DVF to be the magnitude of the velocity, which is the Euclidean distance between the voxels inside the single-cell region and nearest voxel on the closed skeletonized surface. The velocity outside the single-cell regions is set to be 0 to stop the evolution of level set.

\subsubsection{Preliminary results and comparison}

A preliminary result of simulated super-resolution biofilms is shown in Fig. 5.4, where cells that are separated by the improved Bact-3Ds algorithm, are labeled with different colors.
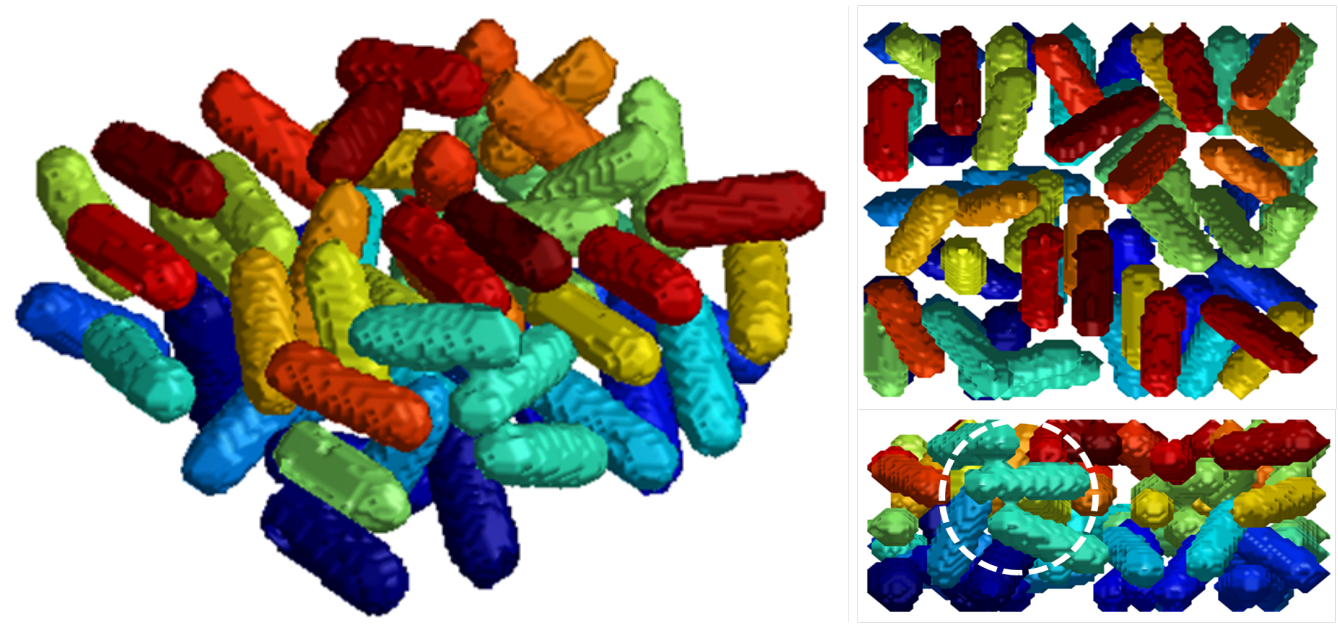

FiguRE 5.4. Preliminary results of Bact-3Ds. Three viewing angles: left: 3D viewpoint; upper right: vertical view; lower right: right side view.

This method currently has an accuracy of $86.7 \%$ by capturing 52 individual bacteria out of 60 (number of the total cells). There are still some cells connected (Fig. 5.4 lower right, circled region). One possible reason for this 
failure is that there are no significant changes of intensity between these cells so that the Hessian approach does not identify the single-cell boundaries.

The improved algorithm shows competitive segmenting results with respect to both Chan-Vese [25] and Bact-3D. Bact-3Ds improves the performance of Bact-3D by roughly three times ( 17 out of 60 individual bacterium is identified using Bact-3D). The segmenting accuracy of Chan-Vese on "randomdistributed" bacteria in 3D is just $1.67 \%$ (1 out of 60 ). Scanned segmenting performance along $z$-direction is shown in Fig. 5.6. 3D reconstructed volume comparison of these three methods is shown in Fig. 5.5.

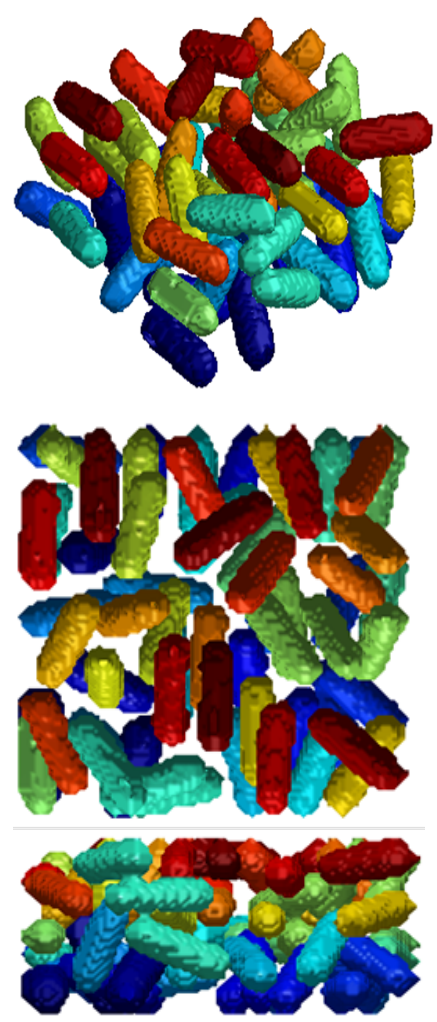

(a) Bact-3Ds
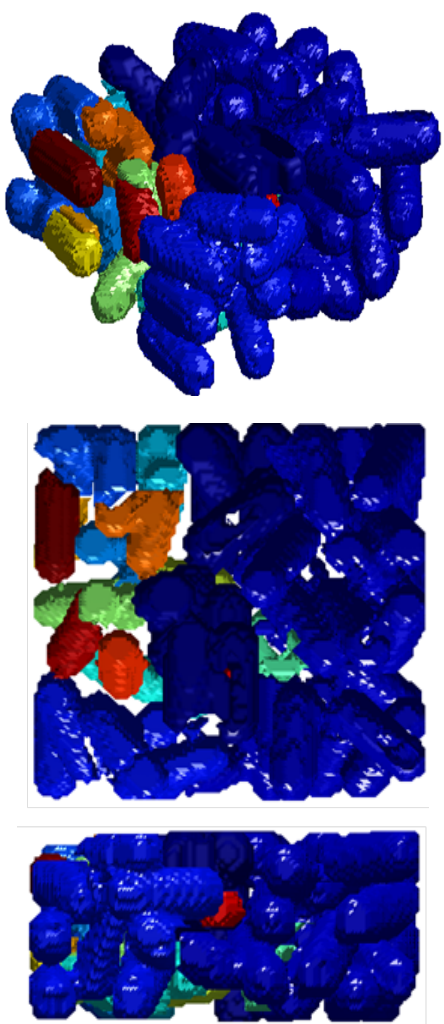

(b)Bact-3D
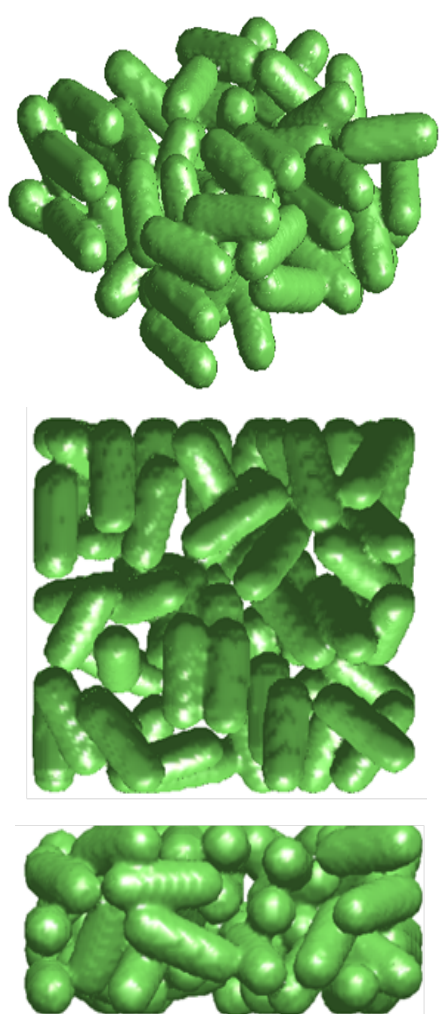

(c) Chan-Vese [25]

FIGURE 5.5. Comparison of improved algorithm with Bact-3D and Chan-Vese [25] in 3D viewpoints. Different colors denote individual components that can be identified. 

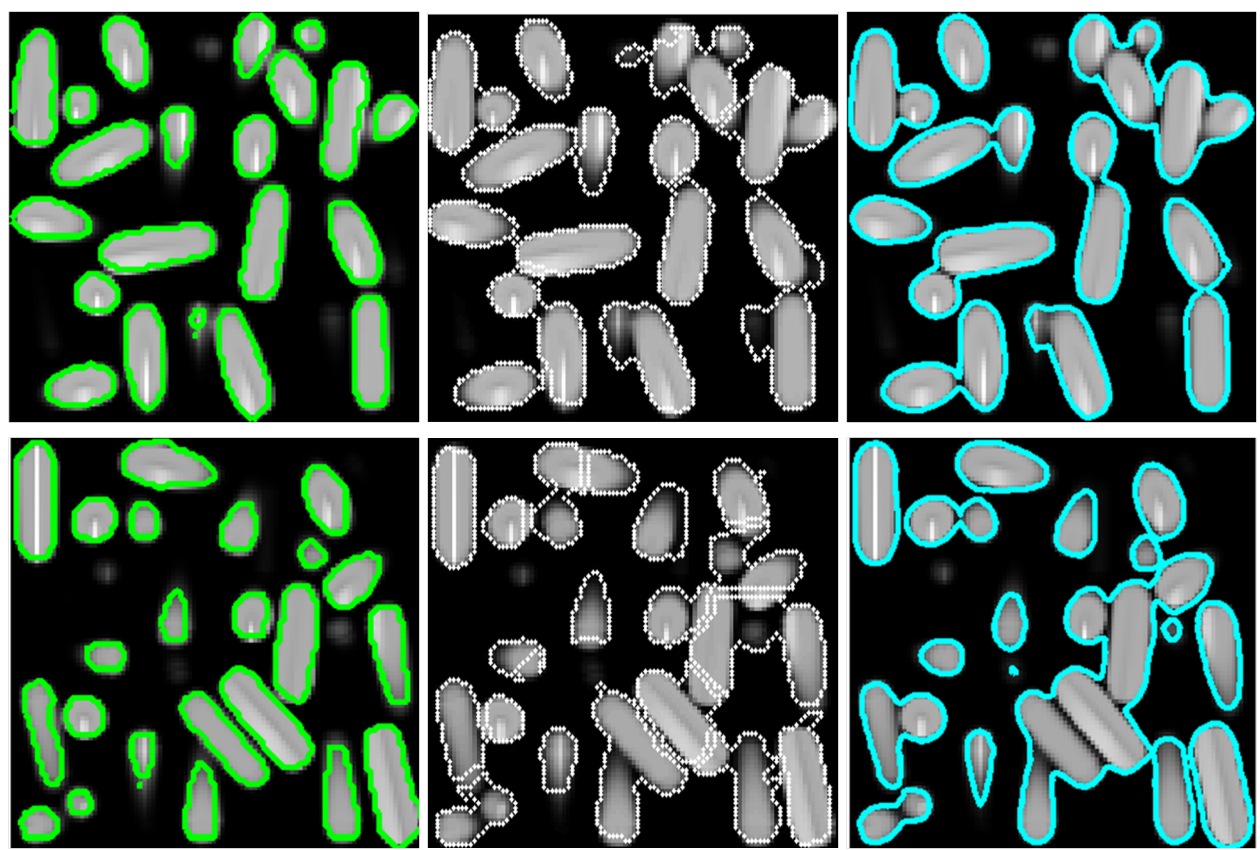

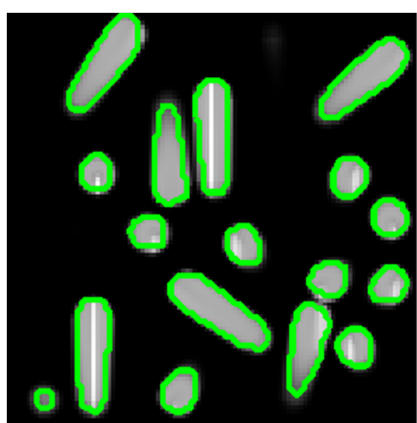

(a) Bact-3Ds

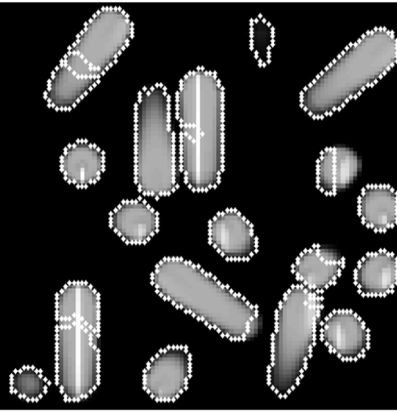

(b)Bact-3D

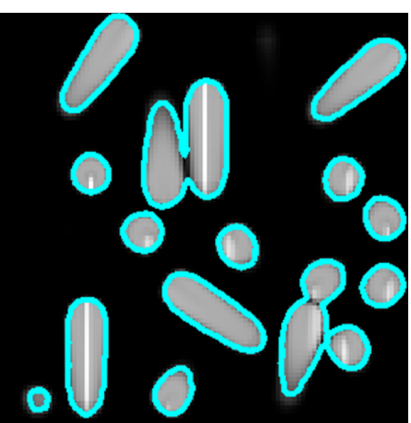

(c) Chan-Vese [25]

FIGURE 5.6. Sliced comparison of improved algorithm with Chan-Vese [25] and Bact-3D.

\subsection{4 discussion}

Although this improved algorithm shows promising results in segmenting simulated "random-distributed" bacterial biofilms in 3D, the model still needs more validation and improvement. Also, we expect further challenges to arise from the analysis of real data. 


\subsection{Future work: from level set to "lsnake"}

Level set segmentation approaches provide us with an efficient model to capture bacterial biofilms; however, the limitations in restricting zero level set size and splitting neighboring overlapping bacteria increase the difficulty of solving this problem.

We are also evaluating a different approach ("lsnake"), which combines the geometric active contour (level set segmentation) with the parametric active contour (snake). This method has two steps. The first step is similar to the distance-based seed selection described in the previous section (sec. 5.1), which takes the advantage of geometric active contour initialization of level set and will not affect the segmentation results. In this case, the process is simplified by selecting the seed for the second step, parametric active contour, in which the initialization of the contour used to be a memory-consuming problem for large 3D datasets. Once the snake is initialized inside each bacterium, a proper force field is needed to attract the contour to the boundary of each bacterium. The benefit of using this second step is that snakes have more flexibility in their structure definition. In addition, two snakes will not merge preventing the situation of cell touching.

Overall, the future work will focus on the numerical implementation of these approaches as well as improving the robustness of the algorithm to tackle actual datasets. 


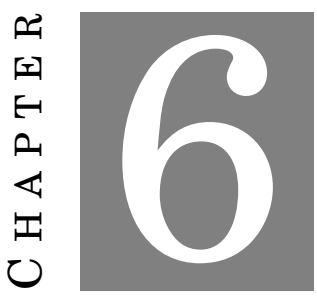

\section{Conclusion}

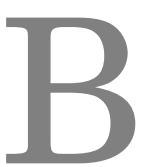

acteria are the most pervasive organisms on earth. To safeguard human from their destructive effect and to harvest the potentials in microbial fuel cell technology, the understanding of the organization within biofilms is crucial.

In this thesis, an effective segmentation technique, Bact-3D, was proposed. It presented a solution to reconstruct micro-level bacterial biofilms, where the images were obtained by novel super-resolution imaging technique. Such a technique overcame the diffraction limit of traditional confocal microscopy. On top of that, it prevented the phototoxicity produced during the traditional imaging process. Bact-3D proposed the concepts of automated layer detection and locally level set segmentation. The skeleton extracted from the smoothed background (the area preclude the ellipse fitting of well-segmented bacteria) ensures the locality of the method. It solved the problem that separation between neighboring cells are difficult to identify over the state-of-the-art. Meanwhile, the trackability of Bact-3D provided the precondition for studying the activities of individual bacterium and their group living habit by offering cell details (size, location and orientation). 
Bact-3D also motivated the improved algorithm, Bact-3Ds, in the preceding chapters. The distance velocity field (DVF) proposed in chapter 5 extended the 2D level set segmentation method used in Bact-3D to 3D local geometric active surface model. It set up single cell regions in 3D to ensure the separation between closely overlapped bacteria, which presented an accuracy of $86.7 \%$ in the preliminary results. We also discussed "lsnake" which would exploit the benefits of both the geometric (level set method) and the parametric (snake) active contour methods to rise to the challenges in complex datasets. 


\section{BIBLIOGRAPHY}

[1] William B. Whitman, David C. Coleman, and William J. Wiebe, "Prokaryotes: The unseen majority," Proceedings of the National Academy of Sciences, vol. 95, pp. 6578-6583, 1998.

[2] H.M. Lappin-Scott and J.W. Costerton, "Microbial biofilms," Biotechnology Research, 2003.

[3] Qiucen Zhang, Guillaume Lambert, David Liao, Hyunsung Kim, Kristelle Robin, Chih-kuan Tung, Nader Pourmand, and Robert H. Austin, "Acceleration of emergence of bacterial antibiotic resistance in connected microenvironments," Science, vol. 333, no. 6050, pp. 1764-1767, 2011.

[4] RM Donlan and JW Costerton, "Biofilms: survival mechanisms of clinically relevant microorganisms," Clinical Microbiology Review, vol. 15, 2002.

[5] B.E Logan, "Exoelectrogenic bacteria that power microbial fuel cells," Nature Reviews Microbiology, vol. 7, pp. 375-381, 2009.

[6] B.E Logan and K Rabaey, "Conversion of wastes into bioelectricity and chemicals by using microbial electrochemical technologies," Science, vol. 337, pp. 686-690, 2012.

[7] Remis JP, Wei D, Gorur A, Zemla M, Haraga J, Allen S, Witkowska HE, Costerton JW, Berleman JE, and Auer M, "Bacterial social networks: structure and composition of myxococcus xanthus outer membrane vesicle chains," Environ Microbiol, vol. 16, no. 2, pp. 598-610, 2014. 
[8] “en.wikipedia.org/wiki/bacterialcellstructure," Wikipedia on bacterial cell structure (online source).

[9] James Gallagher, "http://www.bbc.com/news/health-34392522," BBC news (online), 2015.

[10] Peter Raven, Kenneth Mason, Jonathan Losos, and Susan Singer, "https://commons.wikimedia.org/w/index.php?curid=44194140," Biology 10e Textbook.

[11] Jeff Errington, "Bacterial morphogenesis and the enigmatic mreb helix," Nature Reviews Microbiology, vol. 13, pp. 241-248, 2015.

[12] Sina Wäldchen, Julian Lehmann, Teresa Klein, Sebastian van de Linde, and Markus Sauer, "Light-induced cell damage in live-cell superresolution microscopy," Scientific Reports, vol. 5, 2015.

[13] Veysel Berk, Jiunn C. N. Fong, Graham T. Dempsey, Omer N. Develioglu, Xiaowei Zhuang, Jan Liphardt, Fitnat H. Yildiz, and Steven Chu, "Molecular architecture and assembly principles of vibrio cholerae biofilms," Science, vol. 337, pp. 236-239, 2012.

[14] Marissa K. Lee, Prabin Rai, Jarrod Williams, Robert J. Twieg, and W. E. Moerner, "Small-molecule labeling of live cell surfaces for threedimensional super-resolution microscopy," Journal of the American Chemical Society, vol. 136, pp. 14003,àí14006, 2014.

[15] Bi-Chang Chen, Wesley R. Legant, Kai Wang, Lin Shao, Daniel E. Milkie, Michael W. Davidson, Chris Janetopoulos, Xufeng S. Wu, John A. Hammer III, Zhe Liu, Brian P. English, Yuko Mimori-Kiyosue, Daniel P. Romero, Alex T. Ritter, Jennifer Lippincott-Schwartz, Lillian Fritz-Laylin, R. Dyche Mullins, Diana M. Mitchell, Joshua N. Bembenek, Anne-Cecile Reymann, Ralph BÖhme, Stephan W. Grill, Jennifer T. Wang, Geraldine Seydoux, U. Serdar Tulu, Daniel P. Kiehart, and Eric Betzig, "Lattice 
light-sheet microscopy: Imaging molecules to embryos at high spatiotemporal resolution," Science, vol. 346, no. 6208, pp. 1257998, 2014.

[16] Dong Li, Lin Shao, Bi-Chang Chen, Xi Zhang, Mingshu Zhang, Brian Moses, Daniel E. Milkie, Jordan R. Beach, John A. Hammer III, Mithun Pasham, Tomas Kirchhausen, Michelle A. Baird, Michael W. Davidson, Pingyong $\mathrm{Xu}$, and Eric Betzig, "Extended-resolution structured illumination imaging of endocytic and cytoskeletal dynamics," vol. 349 , no. 6251 , pp. aab3500, 2015.

[17] Scott T Acton and Nilanjan Ray, "Biomedical image analysis: Segmentation," Synthesis Lectures on Image, Video, and Multimedia Processing, vol. 4, no. 1, pp. 1-108, 2009.

[18] Krishna Kant Singh and Akansha Singh, "A study of image segmentation algorithms for different types of images," International Journal of Computer Science Issues, vol. 7, pp. 1694-0814, 2010.

[19] N. Otsu, "A thresholding selection method from gray level histogram," IEEE Trans. Syst. Man. Cybern., vol. SMC-9, no. 1, pp. 62-66, 1979.

[20] "Region and edge based segmentation," http://www.uio.no/studier / emner/matnat/ifi/INF4300 / h11 / undervisningsmateriale / INF43002011-f04-segmentation.pdf.

[21] Tony Lindeberg and Meng-Xiang Li, "Segmentation and classification of edges using minimum description length approximation and complementary junction cues," Computer Vision and Image Understanding., vol. 67, no. 1, pp. 88-89, 1997.

[22] R. Nock and F. Nielsen, "Statistical region merging," IEEE Transactions on Pattern Analysis and Machine Intelligence, vol. 26, no. 11, pp. 14521458, 2004. 
[23] L. Vincent and P. Soille, "Watersheds in digital spaces: an efficient algorithm based on immersion simulations," IEEE Transactions on Pattern Analysis and Machine Intelligence, vol. 13, no. 6, pp. 583-598, 1991.

[24] Tobias Heimann and Hervè Delingette, "Model-based segmentation," Deserno, Thomas Martin. Biomedical Image Processing, pp. 279-303, 2011.

[25] T. F. Chan and L. A. Vese, "Active contours without edges," IEEE Transactions on Image Processing, vol. 10, no. 2, pp. 266-277, 2001.

[26] M. Kass, A. Witkin, and D. Terzopoulos, "Snakes: Active contour models," International Journal of Computer Vision, pp. 321-331, 1988.

[27] Jianbo Shi and Jitendra Malik, "Normalized cuts and image segmentation," IEEE Transactions on Pattern Analysis and Machine Intelligence, vol. 22 , no. 8 , pp. 888-905, 2000.

[28] A. Levinshtein, A. Stere, K. N. Kutulakos, D. J. Fleet, S. J. Dickinson, and K. Siddiqi, "Turbopixels: Fast superpixels using geometric flows," IEEE Transactions on Pattern Analysis and Machine Intelligence, vol. 31, no. 12, pp. 2290-2297, 2009.

[29] Erik Meijering, "Cell segmentation: 50 years down the road," IEEE Signal Processing Magazine, vol. 29, no. 5, pp. 140-145, 2012.

[30] S. Mukherjee and S. T. Acton, "Region based segmentation in presence of intensity inhomogeneity using legendre polynomials," IEEE Signal Processing Letters, vol. 22, no. 3, pp. 298-302, March 2015.

[31] X. Han, C. Xu, and J.L. Prince, "A topology preserving level set method for geometric deformable models," IEEE Transactions on Pattern Analysis and Machine Intelligence, vol. 25, no. 6, pp. 755-768, June 2003.

[32] C. Xu and J. L. Prince, "Snakes, shapes, and gradient vector flow," IEEE Transactions on Image Processing, vol. 7, no. 3, pp. 359-369, 1998. 
[33] R. Sarkar, S. Mukherjee, and S. T. Acton, "Dictionary learning level set," IEEE Signal Processing Letters, vol. 22, no. 11, pp. 2034-2038, 2015.

[34] Bing $\mathrm{Li}$ and Scott T. Acton, "Automatic active model initialization via poisson inverse gradient," IEEE Transactions on Image Processing, vol. 17, pp. 1406-1420, 2008.

[35] Bing Li and Scott T. Acton, "Active contour external force using vector field convolution for image segmentation," IEEE Transactions on Image Processing, vol. 16, no. 8, pp. 2096-2106, 2007.

[36] S. Osher and J.A Sethian, "Fronts propagating with curvature dependent speed: Algorithms based on hamilton-jacobi formulations," Journal of Computational Physics, vol. 79, pp. 12-49, 1988.

[37] X. Bai, C. Sun, and F. Zhou, "Splitting touching cells based on concave points and ellipse fitting," Pattern Recognition, vol. 42, pp. 2434-2446, 2009 .

[38] A. W. Fitzgibbon, M. Pilu, and R. B. Fisher, "Direct least squares fitting of ellipses," 1996.

[39] Florian Levet, Eric Hosy, Adel Kechkar, Corey Butler, Anne Beghin, Daniel Choquet, and Jean-Baptiste Sibarita, "Sr-tesseler: a method to segment and quantify localization-based super-resolution microscopy," 2015 .

[40] S. K. Sadanandan, Ö. Baltekin, K. E. G. Magnusson, , A. Boucharin, P. Ranefall, J. Jaldèn, J. Elf, and C. Wählby, "Segmentation and trackanalysis in time-lapse imaging of bacteria," IEEE Journal of Selected Topics in Signal Processing, vol. 10, no. 1, pp. 174-184, 2016.

[41] J. Yan, A.G. Sharo, H. A. Stone, N. S. Wingreen, and B. L. Bassler, "Vibrio cholerae biofilm growth program and architecture revealed by single-cell live imaging," Proceedings of the National Academy of Sciences, vol. 113, no. 36, pp. E5337-E5343, 2016. 
[42] K. Siddiqi, S. Bouix, A. Tannenbaum, and S. W. Zucker, "The hamiltonjacobi skeleton," Proceedings of the Seventh IEEE International Conference on Computer Vision, 1999.

[43] J. A. Thorpe, "Elementary topics in differential geometry," 1979.

[44] C.O. Solorzano, R. Malladi, S.A. Lelievre, and S.J. Lockett, "Segmentation of nuclei and cells using membrane related protein markers," Journal of Microscopy, vol. 201, pp. 404-415, 2001.

[45] Y. Zhang, B. J. Matuszewski, L. K. Shark, and C. J. Moore, "Medical image segmentation using new hybrid level-set method," pp. 71-76, 2008.

[46] Scott T Acton and Nilanjan Ray, "Biomedical image analysis: tracking," Synthesis Lectures on Image, Video, and Multimedia Processing, vol. 2, no. 1, pp. 1-152, 2006. 